\title{
Globular cluster candidates in the Galactic bulge: Gaia and VVV view of the latest discoveries
}

\author{
F. Gran ${ }^{1,2}$, M. Zoccali ${ }^{1,2}$, R. Contreras Ramos ${ }^{1,2}$, E. Valenti ${ }^{3,4}$, A. Rojas-Arriagada ${ }^{1,2}$, J. A. Carballo-Bello ${ }^{1}$, \\ J. Alonso-García ${ }^{6,2}$, D. Minniti ${ }^{2,5,7}$, M. Rejkuba ${ }^{3,8}$, and F. Surot ${ }^{1}$ \\ ${ }^{1}$ Instituto de Astrofísica, Av. Vicuna Mackenna 4860, Santiago, Chile \\ e-mail: fegran@uc.cl \\ 2 Instituto Milenio de Astrofísica, Santiago, Chile \\ 3 European Southern Observatory, Karl Schwarzschild-Strabe 2, 85748 Garching bei Munchen, Germany \\ ${ }^{4}$ Excellence Cluster ORIGINS, Boltzmann-Strasse 2, 85748 Garching bei München, Germany \\ 5 Departamento de Ciencias Físicas, Facultad de Ciencias Exactas, Universidad Andrés Bello, Av. Fernández Concha 700, \\ Las Condes, Santiago, Chile \\ ${ }^{6}$ Centro de Astronomía (CITEVA), Universidad de Antofagasta, Av. Angamos 601, Antofagasta, Chile \\ 7 Vatican Observatory, 00120 Vatican City State, Italy \\ 8 Excellence Cluster Universe, Boltzmannstr. 2, 85748 Garching, Germany
}

Received 27 December 2018 / Accepted 14 April 2019

\begin{abstract}
Context. Thanks to the recent wide-area photometric surveys, the number of star cluster candidates have risen exponentially in the last few years. Most detections, however, are based only on the presence of an overdensity of stars in a given region or an overdensity of variable stars, regardless of their distance. As candidates, their detection has not been dynamically confirmed. Therefore, it is currently unknown how many and which of the published candidates are true clusters and which are chance alignments.

Aims. We present a method to detect and confirm star clusters based on the spatial distribution, coherence in motion, and appearance on the color-magnitude diagram. We explain and apply this approach to one new star cluster and several candidate star clusters published in the literature.

Methods. The presented method is based on data from the second data release of Gaia complemented with data from the VISTA Variables in the Vía Láctea survey for the innermost bulge regions. This method consists of a nearest neighbors algorithm applied simultaneously over spatial coordinates, star color, and proper motions to detect groups of stars that are close in the sky, move coherently, and define narrow sequences in the color-magnitude diagram, such as a young main sequence or a red giant branch.

Results. When tested in the bulge area $(-10<\ell(\mathrm{deg})<+10 ;-10<b(\mathrm{deg})<+10)$ the method successfully recovered several known young and old star clusters. We report in this work the detection of one new, likely old star cluster, while deferring the others to a forthcoming paper. Additionally, the code has been applied to the position of 93 candidate star clusters published in the literature. As a result, only two of these clusters are confirmed as coherently moving groups of stars at their nominal positions.
\end{abstract}

Key words. surveys - stars: kinematics and dynamics - Galaxy: bulge - globular clusters: general - proper motions

\section{Introduction}

Star clusters are invaluable astrophysics tools for a number of reasons. In addition to being a laboratory for stellar evolution, including chemical evolution and self-enrichment, they are among the few objects for which a rather precise age can be measured. If young and massive, their mass function closely resembles the initial mass function (Leigh et al. 2012; Webb $\&$ Leigh 2015). For all the others, the radial variation of the present-day mass function allows us to quantify the internal dynamical evolution, while the global present-day mass function is related to the dynamical interaction of the cluster with its environment. Although we do not know exactly how and where massive clusters form (Forbes et al. 2018), we know that stars do not form in isolation. Most of them form in groups, if not in massive clusters, and therefore the struggle to get a census of the cluster population of a galaxy is motivated by their relevance for building up the field star population (Kruijssen et al. 2018).
In recent decades, the number of candidate star clusters reported in the literature has increased significantly, thanks to wide area photometric surveys that allowed to literally scan the sky in search of groups (Koposov et al. 2007, 2017; Belokurov et al. 2010, 2014; Muñoz et al. 2012; Ortolani et al. 2012; Laevens et al. 2014, 2015a,b; Bechtol et al. 2015; Kim \& Jerjen 2015; Luque et al. 2017; Ryu \& Lee 2018, and references therein). In the direction of the Galactic bulge only, from VISTA Variables in the Vía Láctea (VVV; Minniti et al. 2010) survey images, Minniti et al. (2011) reported the discovery of the candidate globular cluster (GC) VVV-CL001, Moni Bidin et al. (2011) identified two more candidate clusters CLO02 and CL003, Borissova et al. (2014) listed 58 new infrared star cluster candidates, and another cluster candidate, already catalogued as cluster candidate, was further analyzed in search for variables by Minniti et al. (2017a). Another 84 old cluster candidates were reported by Minniti et al. (2017b,c), and Minniti et al. (2017d) based on detection of spatial overdensities, projected overdensities of RR Lyrae variables, and projected overdensities of RR Lyrae and type II Cepheids, 
respectively. Finally, another five GC candidates were identified by Camargo (2018) by visual inspection Wide-field Infrared Survey Explorer (WISE) images.

Although new star clusters can be initially identified as overdensities, the only way to confirm their cluster nature is to verify that their stars move coherently in space, i.e., they are gravitationally bound. This can be done by measuring either radial velocities or proper motions (PMs) of stars in a region centered at the center of the spatial overdensity. In the present paper, we describe a method to identify unusual concentrations of stars simultaneously in the plane of the sky, vector point diagram (VPD), and color-magnitude diagram (CMD).

The data used in this work come mostly from the second data release (DR2) of the Gaia mission (Gaia Collaboration 2016; Brown et al. 2018), including positions, PMs (Lindegren et al. 2018), and magnitudes in three photometric bands for all of the stars (Riello et al. 2018; Evans et al. 2018). The work of Pancino et al. (2017), Gaia Collaboration (2018), and Vasiliev (2019) illustrates the potential of Gaia to characterize the GCs known up to date. In the region close to the Galactic plane, at latitudes $|b|<3^{\circ}$ the Gaia catalog is highly incomplete owing to the large interstellar extinction affecting optical fluxes, and, to a minor extent, to the higher stellar surface density coupled with the limited transmission bandpass of the satellite. In this region, Gaia detects almost exclusively the brightest blue disk stars, while it is virtually blind to the bulge red giants. On the contrary, the near-infrared (near-IR) VVV observations are optimized for the reddest bulge giants, and the PM catalogs obtained with the method described in Contreras Ramos et al. (2017) are both deeper and more precise than the Gaia catalog. We use the VVV PMs, in addition to Gaia to analyze candidate clusters at latitudes $|b|<3^{\circ}$.

The paper is organized as follows: Sect. 2 describes the automated method to detect - and simultaneously confirm - new star clusters, including the detection of a new old star cluster labeled Gran 1. Section 4 presents an analysis of the PM of stars within 1 arcmin from the nominal position of a sample of 93 old cluster candidates reported by Minniti et al. (2011, 2017b,c,d), Moni Bidin et al. (2011), Camargo (2018), and Bica et al. (2018). The present clustering method was then applied, with relaxed parameters, within a region of 2 arcmins across the center of each cluster candidate, to double check against possible errors in the candidate estimated centers. Finally, Sect. 5 summarizes our results. The VPDs and CMDs of all the unconfirmed candidate clusters are included in Appendix A.

\section{Method to detect coherent groups}

The region selected to search for star clusters was the whole bulge area within $-10^{\circ} \leq(\ell, b) \leq 10^{\circ}$. This region was divided in small circles of $0.8^{\circ}$ radius, centered on every integer degree in Galactic latitude and longitude. An algorithm was developed to search for stars that have an unusually large number of neighbors in a five-dimensional phase-space including coordinates, color, and PMs. For each star with complete information, the algorithm counts the number of neighbors within 1 arcmin in space, one mas/yr in PM, and $1 \mathrm{mag}$ in color using the K-Dimensional Tree (KDTree) implementation on scikit-learn (Pedregosa et al. 2011). For each given field, a minimum threshold of 10 neighbors per star was imposed to ensure statistical significance of the results. Stars with more than 10 neighbors within the complete magnitude range were then searched for groups, in the phase-space mentioned above, with the Density-Based Spatial Clustering of Applications with Noise (DBSCAN) algorithm
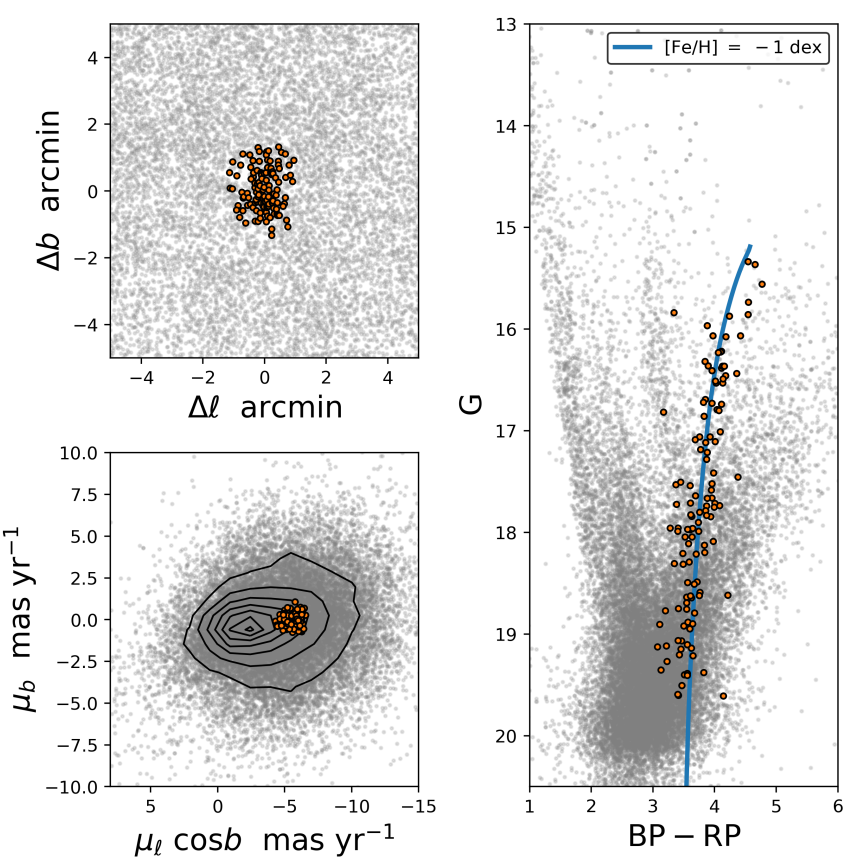

Fig. 1. Globular cluster Terzan 1 as detected by our algorithm. The orange points represent the detected overdensity along with background stars (gray points) within 15 arcmins from the detected cluster centroid. The gray stars define three populated sequences from left to right: the disk MS, disk red clump (both spread along the line of sight), and the bulge upper red giant branch with a PGPUC isochrone overplotted.

(Ester et al. 1996) also implemented in the scikit-learn package. Groups were kept as candidate clusters and then visually inspected only if they contained at least 20 members. We note that a group of neighbor stars define "a neighborhood" that may end up being significantly larger than the phase-space radius (1 arcmin, $1 \mathrm{mas} \mathrm{yr}^{-1}, 1 \mathrm{mag}$ ) used for the initial search around each star.

It should be noted that several false positives are detected by the algorithm, that is, close to the mean PM of bulge $(-6,-0.2)$ and disk $(-2,-0.5)$ field stars, in the VPD. After visual inspection, however, we keep only clusters that define narrow sequences in the CMD that are significantly different, by shape and/or tightness, from the main branches of the bulge+disk CMD.

A very first step for the validation of the algorithm described above is that it must be able to rediscover all the clusters known to exist in the area explored. In order to verify this, the catalog of overdensities was cross-matched with the latest version of the Global survey of Milky Way star clusters (MWSC; Kharchenko et al. 2016) catalog to identify all the clusters that were previously known. Indeed, we detected all the 45 known bulge GCs in this region and $17 \%$ (22/129) of the open clusters up to 5 arcmins from their nominal center. Five known GCs located in the region $|b|<2.5^{\circ}$ (Terzan 4, Terzan 6, Terzan 10, 2MASS-GC002, and Djorg 2) were not detected in Gaia, but they were detected in VVV. Another 4 that are listed in the MWSC catalog as candidate GCs, namely ESO 456-09, ESO 373-12, FSR 0019, and FSR 0025, were not detected in either of the two surveys. Based on our data, we do not find any evidence allowing us to confirm their cluster nature.

Figure 1 shows the detection of Terzan $1\left(\ell=-2.44^{\circ}, b=\right.$ $0.99^{\circ}$, Ortolani et al. 1999). The mean PM of this cluster is very similar to the mean PM of bulge stars (lower left panel). Nonetheless, the algorithm detects a higher concentration of 

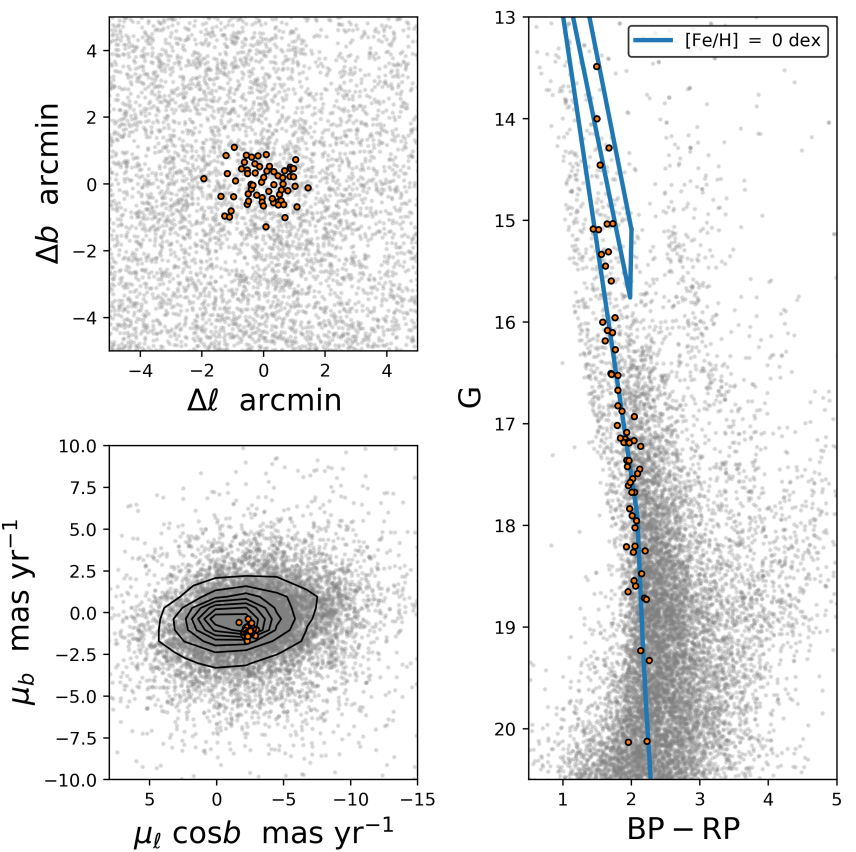

Fig. 2. Open cluster ESO 589-26 as detected by our algorithm. The background sequences are the same as in Fig. 1, although the bulge red giant branch is highly incomplete because this cluster is located very close to the Galactic plane $\left(b=0.33^{\circ}\right)$ in a region of the sky with large interstellar extinction.

stars, in the VPD, with respect to a smoother background. Of course, the detection is also triggered by the clustering of Terzan 1 stars both in the plane of the sky (upper left) and in the CMD (right). We note that, as explained above, the algorithm can detect compact sequences of stars, in addition to roundish groups. We overplot a PGPUC isochrone (Valcarce et al. 2013) for a $[\mathrm{Fe} / \mathrm{H}]=-1 \mathrm{dex}, 12 \mathrm{Gyr}$ simple stellar population, at the cluster distance quoted in Kharchenko et al. (2016), to confirm that the overdensity is indeed Terzan 1 .

The algorithm is also able to detect open star clusters. As an example, Fig. 2 shows the recovery of ESO-589-26, a young star cluster reported at $\mathrm{RA}=18: 02: 14 \mathrm{Dec}=-21: 54: 54$ $(l, b)=\left(7.9517^{\circ}, 0.3279^{\circ}\right)$, which our code independently detected 7 arcsec away from its nominal position. A total of 62 cluster members were identified with our method, defining a narrow main sequence in the CMD and a very coherent group in the VPD. A 3 Myr PARSEC (Marigo et al. 2017) isochrone of solar metallicity is included in the CMD to guide the eye. The isochrone was shifted to a distance of $\sim 2.4 \mathrm{kpc}$, as reported in Kharchenko et al. (2016).

Application of this neighbor algorithm to the selected bulge area yielded several cluster candidates not listed in the MWSC catalog. We visually inspected the region within a radius of 5 arcmins from the center of each of these cluster candidates and ended up with at least seven new GCs. The new clusters will be published in dedicated forthcoming papers once they have been spectroscopically confirmed. We show in this paper only one of the new GCs, which we name Gran 1, as an example of the ability of the algorithm to find new clusters. Further, we examine the candidate cluster VVV-CL001 (Minniti et al. 2011), VVVCL002, VVV-CL003, and VVV-CL004 discussed in Moni Bidin et al. (2011), the 84 candidate clusters published in the series of papers by Minniti et al. (2017b,c), and Minniti et al. (2017d), plus another five candidates presented in Camargo (2018), one of which is further analyzed by Bica et al. (2018).
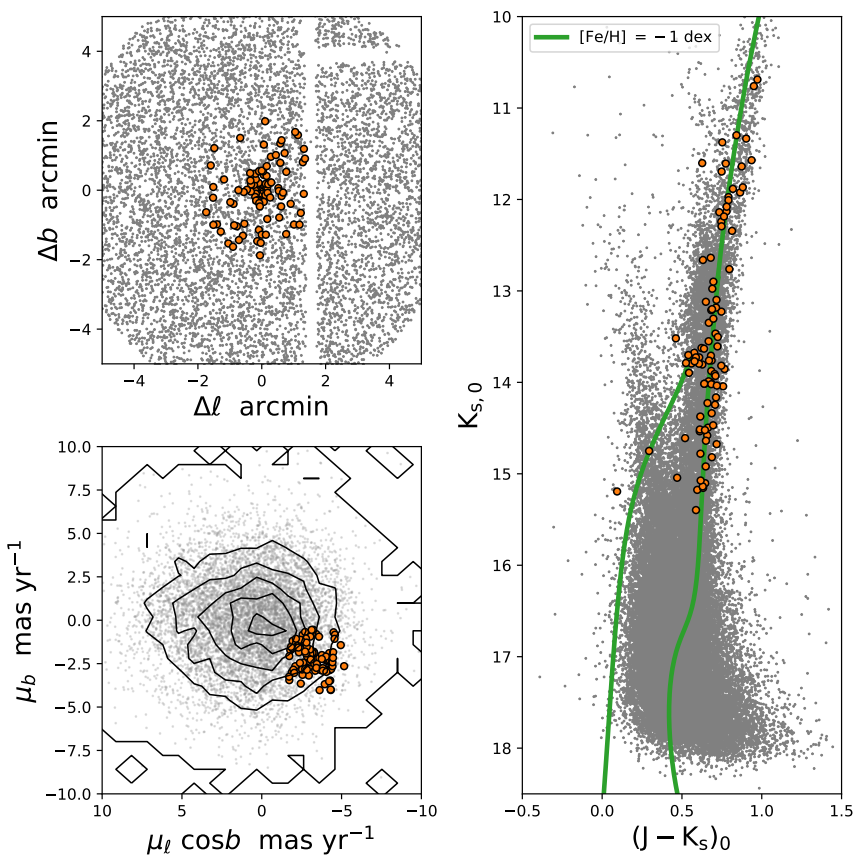

Fig. 3. New GC Gran 1 as detected in the plane of the sky (upper left), in the VPD (lower left), and in the dereddened CMD (right). All data are from VVV, using the dereddened PSF photometry by Surot et al. (2019) and the PMs from Contreras Ramos et al. (2017). Small, light gray dots are field stars within 5 arcmin from the cluster center given in Table 1, while large orange dots are bona fide cluster members. Blank stripes in the upper left panel denote the chip separations where stars were rejected because the PM values were not well constrained (Contreras Ramos et al. 2017).

\section{New globular cluster Gran 1}

A new cluster, not present in the MWSC catalog, was initially detected as a group of 24 stars within 0.731 arcmins from $(\ell, b)=$ $\left(-1.22^{\circ},-3.98^{\circ}\right)(\mathrm{RA}=17: 58: 36.61 \mathrm{Dec}=-32: 01: 10.72)$. As we believe that this is the first detection of a cluster in this position, we name it Gran 1. The plots that triggered its discovery are shown in Fig. 3. The 24 clustered stars initially found by the code were used to define a cluster center, both in the plane of the sky and in the VPD. These positions were used to select all the stars included both within 2 arcmin in the sky and within 2 mas $^{-1} r^{-1}$ in the VPD, which yielded 95 stars, shown as filled orange circles in Fig. 3. The CMD in the right panel demonstrates that they are compatible with the RGB and horizontal branch (HB) of a cluster with $[\mathrm{Fe} / \mathrm{H}]=-1 \mathrm{dex}$, located at a distance of $\approx 8.8 \mathrm{kpc}$, or $\mathrm{m}-\mathrm{M}+\mathrm{A}_{K_{\mathrm{s}}}=15 \mathrm{mag}$, i.e., within the Galactic bulge. We emphasize that the cluster metal content, distance, and age cannot be constrained by the present data, as the turnoff cannot be identified in the CMD, future spectroscopic follow-up will derive more precise parameters of this cluster. As a reference, we show a 12 Gyr PGPUC isochrone overplotted to the data, using the putative red clump and HB stars $\left(J-K_{\mathrm{s}} \sim 0.25 \mathrm{mag}\right.$ and $K_{\mathrm{s}} \sim 15 \mathrm{mag}$.) as an anchor to estimate a reddening of $E\left(J-K_{\mathrm{s}}\right)$ and, adopting the extinction law by Nishiyama et al. (2009), a total extinction of $A_{K_{\mathrm{s}}}$. The age was assumed to be larger than $10 \mathrm{Gyr}$, owing to the presence of the two blue HB stars, whose nature would need confirmation. If these two stars are proved to be field stars, the age of the candidate cluster could be lower than the estimate we give in Table 1.

It should be noted that Gran 1 would not have been discovered as a high spatial concentration only because this cluster has 
Table 1. Basic parameters for the newly discovered GC Gran 1, derived from the present analysis.

\begin{tabular}{lcc}
\hline \hline Parameter & Value & Unit \\
\hline$\ell$ & -1.2320 & $\mathrm{deg}$ \\
$b$ & -3.9776 & $\mathrm{deg}$ \\
$\mathrm{RA}(\mathrm{J} 2015.5)$ & $17: 58: 36.61$ & $\mathrm{hh}: \mathrm{mm}: \mathrm{ss}$ \\
$\operatorname{Dec}(\mathrm{J} 2015.5)$ & $-32: 01: 10.72$ & $\mathrm{dd}: \mathrm{mm}: \mathrm{ss}$ \\
$E\left(J-K_{\mathrm{s}}\right)$ & $\sim 0.45$ & $\mathrm{mag}$ \\
$A_{K_{\mathrm{s}}}$ & $\sim 0.24$ & $\mathrm{mag}$ \\
$d_{\odot}$ & $\sim 8.8$ & $\mathrm{kpc}^{-1}$ \\
$\mu_{\ell} \cos b$ & -10.9426 & $\mathrm{mas} \mathrm{yr}^{-1}$ \\
$\mu_{b}$ & 3.0252 & $\mathrm{mas} \mathrm{yr}^{-1}$ \\
$\mu_{\alpha} \cos \delta$ & -8.0583 & $\mathrm{mas} \mathrm{yr}^{-1}$ \\
$\mu_{\delta}$ & -8.0833 & $\mathrm{mas} \mathrm{yr}^{-1}$ \\
$\mathrm{Age}$ & $\sim 8-12$ & $\mathrm{Gyr}$ \\
{$[\mathrm{Fe} / \mathrm{H}]$} & $\sim-1$ & $\mathrm{dex}$ \\
\hline
\end{tabular}

Notes. We emphasize that the metallicity, age, and distance for the cluster are very uncertain, as they were derived using 76 stars by comparing the observed CMD with a PGPUC isochrone.

an overdensity of only 3.4 sigma above the mean stellar density of the field in this region. Gran 1 is detected in this case because the selected cluster member stars are simultaneously clustered in the plane of the sky and they share a coherent motion with a

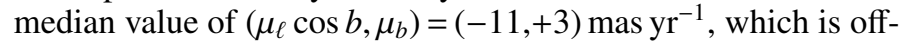
centered with respect to the mean PM of field stars. The integrated magnitude of Gran 1, down to the limit magnitude of the VVV catalog is $K_{\mathrm{s}}=8.07$, while that of Terzan 1 in the same range is $K_{\mathrm{s}}=5.24$. Gran 1 is similar in luminosity to Whiting 1 , AM 4, or Koposov 1 (Harris 2010). A JYZ color image of the newly discovered cluster is shown in Fig. 4.

\section{Application to candidate globular clusters in the literature}

Hereafter we examine various sets of candidate clusters recently published in the literature. Specifically, Minniti et al. (2011) reported on the discovery of a low mass GC, named VVV GC001, at coordinates $(l, b)=\left(5.25^{\circ}, 0.78^{\circ}\right),(\mathrm{RA}=17: 54: 42.5$, Dec $=-24: 00: 53)$, that is, approximately 10 arcmins away from the known GC UKS 1. In the same year, Moni Bidin et al. (2011) detected three new candidate clusters tentatively named VVV GC002, GC003, and GC004. Upon analysis of their CMD, the same authors concluded that they were most likely a GC, a stellar association, and an overdensity whose nature could not be established, respectively.

In 2017, Minniti et al. compiled a catalog containing 84 GC candidates based on overdensities of stars in the plane of the sky (Minni 1 to Minni 21; Minniti et al. 2017b) and on the coincidence in the plane of the sky of a couple or more RR Lyrae (Minni 22 to Minni 60; Minniti et al. 2017c) and/or Type II Cepheids stars (Minni 61 to Minni 84; Minniti et al. 2017d) within 2 arcmins. In the latter two papers, these authors checked that at least two of the variables within each group had magnitudes consistent with similar distances. Finally, Camargo (2018) published a list of 5 clusters visually selected in multicolor images from the WISE satellite, and then analyzed these sources using 2MASS photometry and Gaia DR2 PMs.

The algorithm developed in this work, which were applied to the Gaia PM catalog, did not blindly detect any of the 93 cluster candidates mentioned above (4 VVV GCs, 84 Minnis, plus

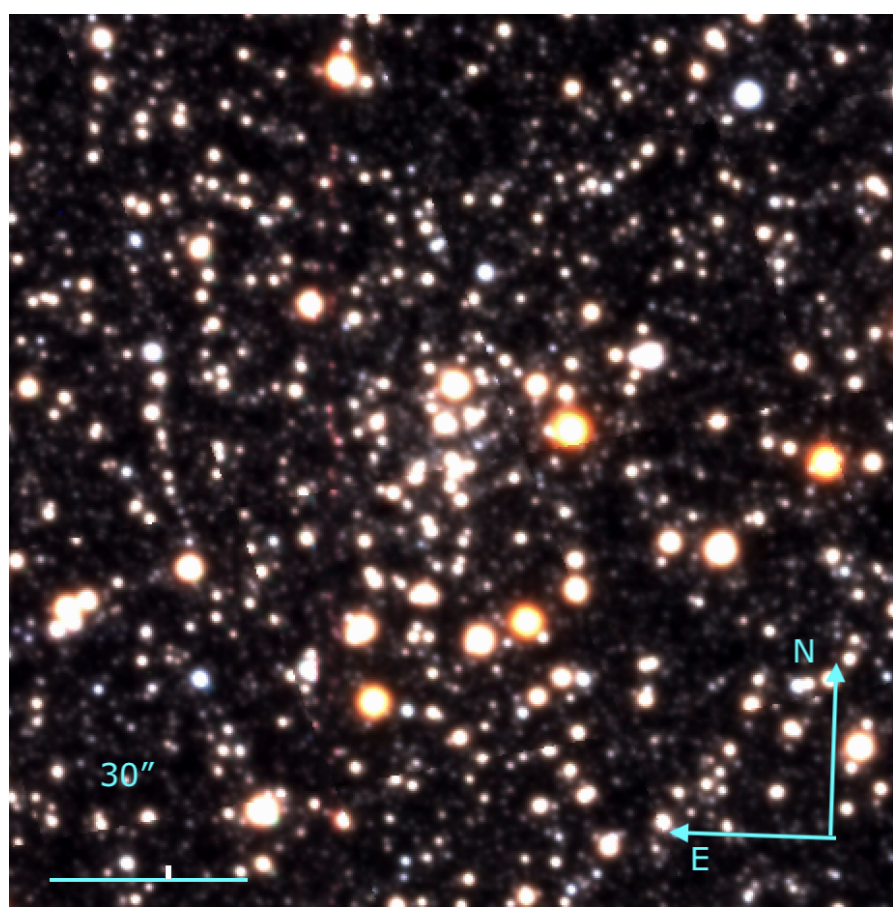

Fig. 4. $J Y Z$ color image of the new GC Gran 1 from the VVV Data Release 4.

5 Camargos) in the Gaia DR2 catalog. Because of the much higher stellar density close to the plane, a blind search across the VVV area within $|b| \lesssim 3^{\circ}$ yielded a large number of overdensities, which will be examined and validated in a forthcoming paper. For the present purpose, we only need to search in a very restricted area around their nominal centers to validate previously published candidate clusters. Therefore, we ran the original code, this time allowing for a lower minimum number of member stars (15 instead of 20) only in a region of 2 arcmins radius around the candidate centers for stars brighter than $G=19$ mag. We ran this restricted search for all the 93 clusters, on the Gaia catalog, and for the 54 candidates (4 VVV GC, 46 Minnis, and 4 Camargos) within $|b| \lesssim 3^{\circ}$ on the VVV PM catalog.

As a result, only the candidates VVV GC001 and GC002 seem to be real clusters. Both of these lie relatively close to the plane, and nothing is detected in the Gaia catalog. In VVV data, however, the algorithm picks up an overdensity of stars whose mean PM is offset with respect to the mean PM of field stars, and who define a rather narrower RGB sequence compared with that of field stars within the same spatial region. This is illustrated in Figs. 5 and 6.

For all the others, the algorithm either did not detect any overdensity nor did it detect a broad peak, but this peak was centered in the VPD at the same position of the median PM of field stars and the CMD did not show anything different from the CMD of field stars. Appendix A shows the spatial selection of stars within 1 arcmin from the candidate center, together with their position in the VPD, compared to that of field stars within 10 arcmins of the published center, and the CMD of both. For each cluster, we show these diagrams from Gaia data on the left and, if available, from VVV data on the right. We also include the two confirmed candidates VVV GC001 and VVV GC002 in order to demonstrate that we would have been able to confirm these even without using the clustering algorithm. In fact, by selecting stars within 1 arcmin from their center in the VVV 

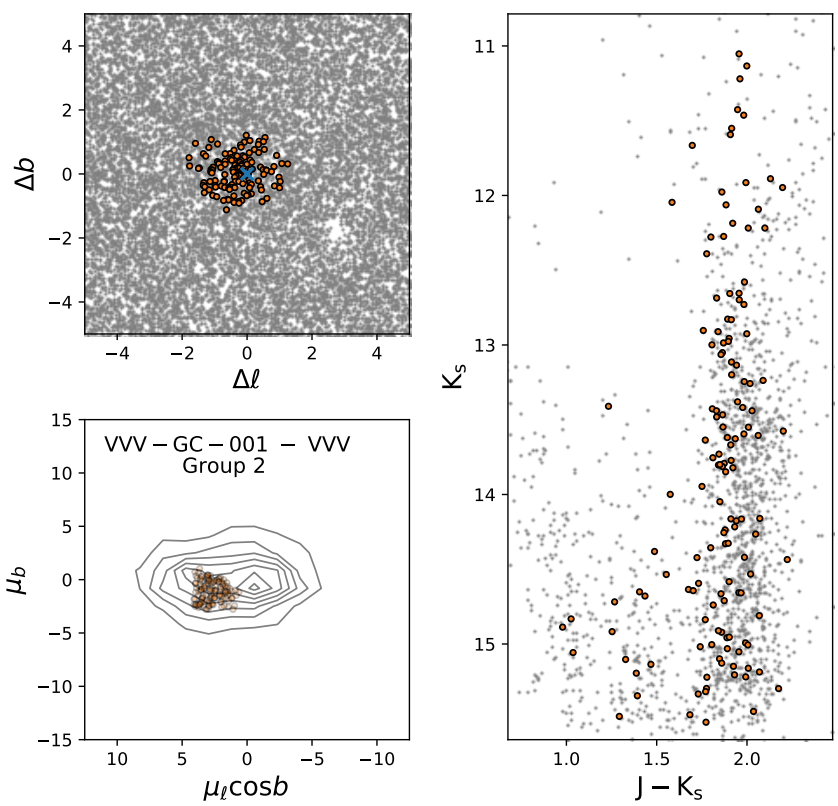

Fig. 5. Diagnostic plots for GC001. Stars within 2 arcmins from the nominal cluster center were used to perform the clustering algorithm. The spatial distribution of stars (Group 2 defined by the code) that comprise the GC are shown in orange points (upper left), plotted in the VPD (bottom left) and in the CMD. We note that the distribution of cluster stars is shifted with respect to the contours of the total distribution of stars in the VPD.

data the field contamination is larger, but a group of stars with a mean PM offset from that of field stars is visible in both of these. The offset is smaller for GC001, where the CMD is better defined; the offset is larger in GC002, where the CMD alone would be more ambiguous. On the contrary, by means of these plots, we reject all the clusters that were not the VPD nor the CMD, which allows us to see a cluster-like structure clearly different from the dominant field population. With this argument, we concluded that by means of the present data we cannot confirm the cluster nature of any of the other 91 candidates.

A few considerations are in order. First, it is expected that by pure Poisson statistics applied to the VPD, we would expect larger fluctuations where the density of stars is higher. That is, at the peak PM of field stars. In other words, we expect a large number of false positives whose mean PMs are identical to those of the dominant population of field stars, whether bulge or disk. Second, we used the catalog of 150 GC PMs provided by Vasiliev (2019), together with the mean bulge PM from Reid \& Brunthaler $(2004)^{1}$ to estimate what fraction of known clusters are expected to have a mean PM centered at the mean value for bulge stars. The result is that only 7 clusters have mean PM within 1 mas yr$^{-1}$ from the mean bulge PM. Of those, only 3 are located within the area explored in this work, where there are in total 49 clusters. In other words, only $7 \%$ of the clusters located in the bulge are expected to have the same mean PM as bulge stars.

If the candidate clusters were massive enough, we would detect these clusters even if their PM would be identical to the peak of bulge stars, as we do for Terzan 1. Therefore, we can

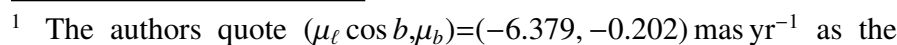
absolute PM of Sagittarius A, which must coincide with the mean absolute PM of bulge stars. Converted to equatorial coordinates, this gives $\left(\mu_{\alpha} \cos \delta, \mu_{\delta}\right)=(-3.15,-5.55)$ mas $_{\mathrm{yr}^{-1}}$, which we subtract from the values in Table B.1 from Vasiliev (2019).
}
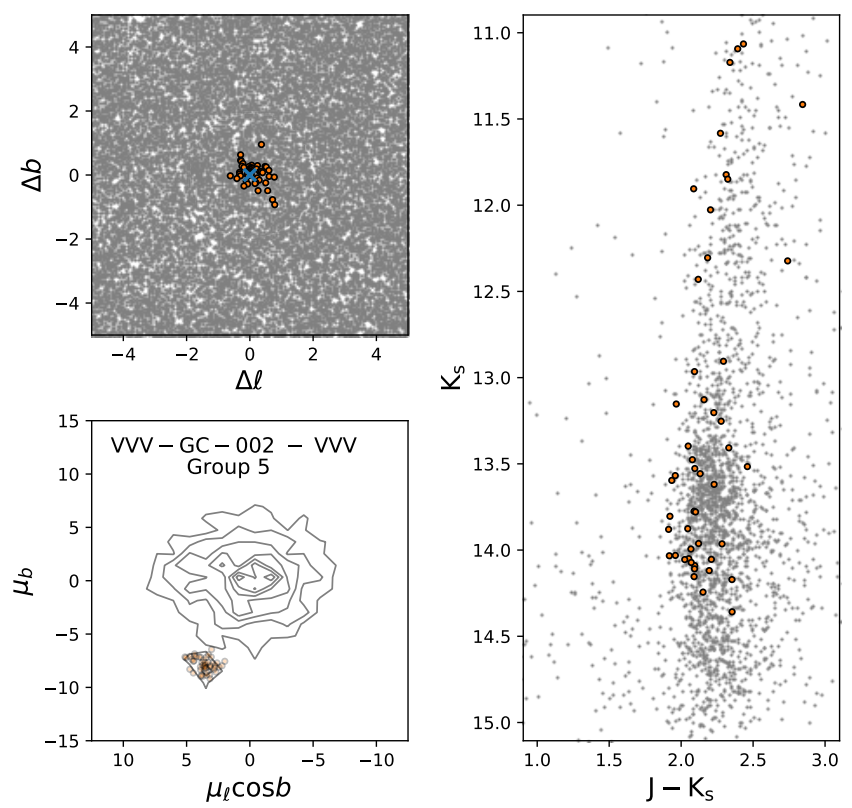

Fig. 6. Same as Fig. 5 for the GC VVV GC002.

safely exclude that any of the 91 candidates that we do not confirm in this work are as massive as Terzan 1. In order for these candidates to be real, they must have a very low mass, and/or low central concentration and their PMs must be identical to those of bulge stars. This is expected to be true for at most 6 of these candidates.

\section{Summary}

We present a clustering algorithm that looks for overdensities simultaneously in a five-dimensional space including the two coordinates in the sky, the corresponding stellar PMs in each coordinate, and one color. The algorithm was independently applied to the Gaia DR2 catalog of a region of the sky within $-10^{\circ} \leq(\ell, b) \leq 10^{\circ}$, and to a VVV catalog including point spread function (PSF) photometry and PMs for stars within $|b|<3^{\circ}$, where Gaia is highly incomplete for bulge stars owing to large interstellar extinction.

With the adopted parameters, discussed in Sect. 2 the algorithm is able to recover all the clusters known to be present in this area and to find several new young and old star clusters. We presented the detection of one old cluster named Gran 1 and deferred the discussion of the other new clusters to forthcoming, dedicated paper.

The PMs of Gaia and VVV were then used to confirm or dismiss the cluster nature of 93 cluster candidates recently presented in the literature. Based on the requirement that the putative cluster members must move coherently, we could confirm 2 of these candidate (VVV GC001 and GC002) and discarded another 91 .

The present result emphasizes that statistical fluctuations of the projected stellar density in the plane of the sky or a chance alignment of a few variable stars can be relatively frequent in a huge survey such as Gaia and VVV. These statistical fluctuations can be easily mistaken for a star cluster for two reasons. First, the stellar population of the Galactic bulge has a (relatively low) spread in metallicity and age that makes its RGB only slightly wider than that of a GC. Second, when selecting stars in a small region of the sky, that is within a few arcmins from a spatial 
overdensity peak, the CMD sequences always appear narrower than those of a wider region because they are affected by a lower differential extinction. For this reason, the list of candidate new star clusters published in the last few years has increased enormously. Only stellar motions, however, can confirm the real cluster nature of a detected overdensity and these have proven to dismiss the large majority of candidates. Given the present availability of Gaia PMs across almost the whole sky, the claimed detection of new candidate clusters should always be supported by kinematics analysis, proving that the cluster member stars move coherently in space.

By means of the present data, we cannot exclude that a few of the candidates are extremely low mass clusters that happen to have zero PM with respect to bulge stars, are located at a distance very close to $8 \mathrm{kpc}$, and present relatively broad CMD sequences possibly owing to differential extinction. We can only state that, because of these characteristics, they would be invisible to both Gaia and VVV. It is important to keep this in mind, however, when deciding whether to allocate telescope time to follow-up studies of such candidates.

Acknowledgements. This work is part of the Ph.D. thesis of F.G., funded by grant CONICYT-PCHA Doctorado Nacional 2017-21171485. F.G. also acknowledges CONICYT-Pasantía Doctoral en el Extranjero 2019-75190166 and ESO SSDF 19/20 (ST) GAR funding. We acknowledge support from the Ministry for the Economy, Development, and Tourism's Programa Iniciativa Científica Milenio through grant IC120009, awarded to Millenium Institute of Astrophysics (MAS), the BASAL CATA Center for Astrophysics and Associated Technologies through grant AFB-170002, and from FONDECYT Regular 1150345. JAC-B acknowledges financial support to CAS-CONICYT 17003. Based on observations taken within the ESO VISTA Public Survey VVV, Program ID 179.B-2002. This work has made use of data from the European Space Agency (ESA) mission Gaia (https://www.cosmos.esa.int/gaia), processed by the Gaia Data Processing and Analysis Consortium (DPAC, https: //www.cosmos.esa.int/web/gaia/dpac/consortium). Funding for the DPAC has been provided by national institutions, in particular the institutions participating in the Gaia Multilateral Agreement. This research made use of: TOPCAT (Taylor et al. 2005), GitHub, IPython (Pérez \& Granger 2007), numpy (van der Walt et al. 2011), matplotlib (Hunter 2007), Astropy, a communitydeveloped core Python package for Astronomy (Astropy Collaboration 2013), scikit-leran Pedregosa et al. (2011), galpy: A Python Library for Galactic Dynamics Bovy (2015), and "Aladin sky atlas" developed at CDS, Strasbourg Observatory, France (Bonnarel et al. 2000; Boch et al. 2014). This research has made use of NASA's Astrophysics Data System.

\section{References}

Astropy Collaboration (Robitaille, T. P., et al.) 2013, A\&A, 558, A33 Bechtol, K., Drlica-Wagner, A., Balbinot, E., et al. 2015, ApJ, 807, 50 Belokurov, V., Walker, M. G., Evans, N. W., et al. 2010, ApJ, 712, L103 Belokurov, V., Irwin, M. J., Koposov, S. E., et al. 2014, MNRAS, 441, 2124 Bica, E., Minniti, D., Bonatto, C., \& Hempel, M. 2018, PASA, 35, e025 Boch, T., \& Fernique, P. 2014, ASP Conf. Ser., 485, 277
Bonnarel, F., Fernique, P., Bienaymé, O., et al. 2000, A\&AS, 143, 33 Borissova, J., Chené, A.-N., Ramírez Alegría, S., et al. 2014, A\&A, 569, A24

Bovy, J. 2015, ApJS, 216, 29

Brown, A. G. A., Vallenari, A., Prusti, T., et al. 2018, A\&A, 616, A1

Camargo, D. 2018, ApJ, 860, L27

Contreras Ramos, R., Zoccali, M., Rojas, F., et al. 2017, A\&A, 608, A140

Ester, M., Kriegel, H. P., Sander, J., \& Xu, X. 1996, A density-based algorithm for discovering clusters in large spatial databases with noise (AAAI Press), 226

Evans, D. W., Riello, M., De Angeli, F., et al. 2018, A\&A, 616, A4

Forbes, D. A., Bastian, N., Gieles, M., et al. 2018, Proc. Roy. Soc. London Ser. A, 474, 20170616

Gaia Collaboration (Prusti, T., et al.) 2016, A\&A, 595, A1

Gaia Collaboration (Helmi, A., et al.) 2018, A\&A, 616, A12

Harris, W. E. 2010, ArXiv e-prints [arXiv:1012 . 3224]

Hunter, J. D. 2007, Comput. Sci. Eng., 9, 90

Kharchenko, N. V., Piskunov, A. E., Schilbach, E., Röser, S., \& Scholz, R. D. 2016, A\&A, 585, A101

Kim, D., \& Jerjen, H. 2015, ApJ, 799, 73

Koposov, S., de Jong, J. T. A., Belokurov, V., et al. 2007, ApJ, 669, 337

Koposov, S. E., Belokurov, V., \& Torrealba, G. 2017, MNRAS, 470, 2702

Kruijssen, J. M. D., Pfeffer, J. L., Reina-Campos, M., Crain, R. A., \& Bastian, N. 2018, MNRAS, 1537

Laevens, B. P. M., Martin, N. F., Sesar, B., et al. 2014, ApJ, 786, L3

Laevens, B. P. M., Martin, N. F., Ibata, R. A., et al. 2015a, ApJ, 802, L18

Laevens, B. P. M., Martin, N. F., Bernard, E. J., et al. 2015b, ApJ, 813, 44

Leigh, N., Umbreit, S., Sills, A., et al. 2012, MNRAS, 422, 1592

Lindegren, L., Hernández, J., Bombrun, A., et al. 2018, A\&A, 616, A2

Luque, E., Pieres, A., Santiago, B., et al. 2017, MNRAS, 468, 97

Marigo, P., Girardi, L., Bressan, A., et al. 2017, ApJ, 835, 77

Minniti, D., Lucas, P. W., Emerson, J. P., et al. 2010, New Astron., 15, 433

Minniti, D., Hempel, M., Toledo, I., et al. 2011, A\&A, 527, A81

Minniti, D., Palma, T., Dékány, I., et al. 2017a, ApJ, 838, L14

Minniti, D., Geisler, D., Alonso-García, J., et al. 2017b, ApJ, 849, L24

Minniti, D., Alonso-García, J., Braga, V., et al. 2017c, Res. Notes Am. Astron. Soc., 1, 16

Minniti, D., Alonso-García, J., \& Pullen, J. 2017d, Res. Notes Am. Astron. Soc., 1,54

Moni Bidin, C., Mauro, F., Geisler, D., et al. 2011, A\&A, 535, A33

Muñoz, R. R., Geha, M., Côté, P., et al. 2012, ApJ, 753, L15

Nishiyama, S., Tamura, M., Hatano, H., et al. 2009, ApJ, 696, 1407

Ortolani, S., Barbuy, B., Bica, E., et al. 1999, A\&A, 350, 840

Ortolani, S., Bonatto, C., Bica, E., Barbuy, B., \& Saito, R. K. 2012, AJ, 144, 147

Pancino, E., Bellazzini, M., Giuffrida, G., \& Marinoni, S. 2017, MNRAS, 467, 412

Pedregosa, F., Varoquaux, G., Gramfort, A., et al. 2011, J. Mach. Learn. Res., 12,2825

Pérez, F., \& Granger, B. E. 2007, Comput. Sci. Eng., 9, 21

Reid, M. J., \& Brunthaler, A. 2004, ApJ, 616, 872

Riello, M., De Angeli, F., Evans, D. W., et al. 2018, A\&A, 616, A3

Ryu, J., \& Lee, M. G. 2018, ApJ, 863, L38

Surot, F., Valenti, E., Hidalgo, S. L., et al. 2019, A\&A, 623, A168

Taylor, M. B. 2005, ASP Conf. Ser., 347, 29

Valcarce, A. A. R., Catelan, M., \& De Medeiros, J. R. 2013, A\&A, 553, A62

van der Walt, S., Colbert, S. C., \& Varoquaux, G. 2011, Sci. Eng., 13, 22

Vasiliev, E. 2019, MNRAS, 484, 2832

Webb, J. J., \& Leigh, N. W. C. 2015, MNRAS, 453, 3278 


\section{Appendix A: Literature GC candidates}
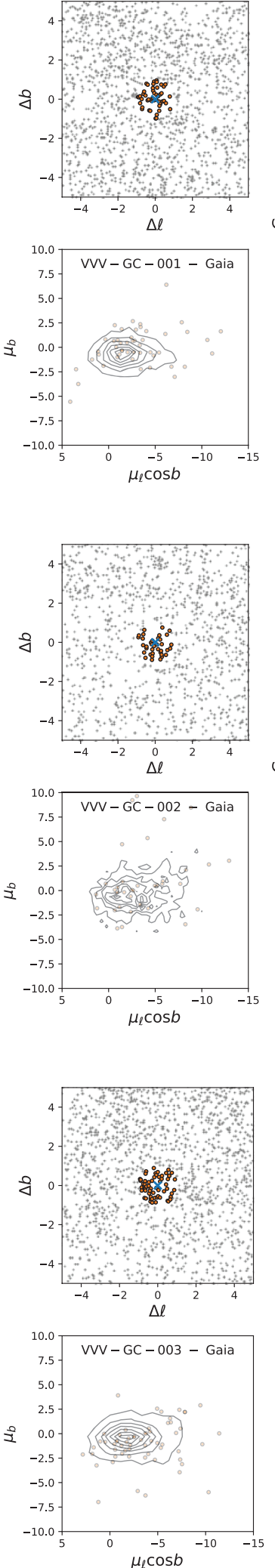
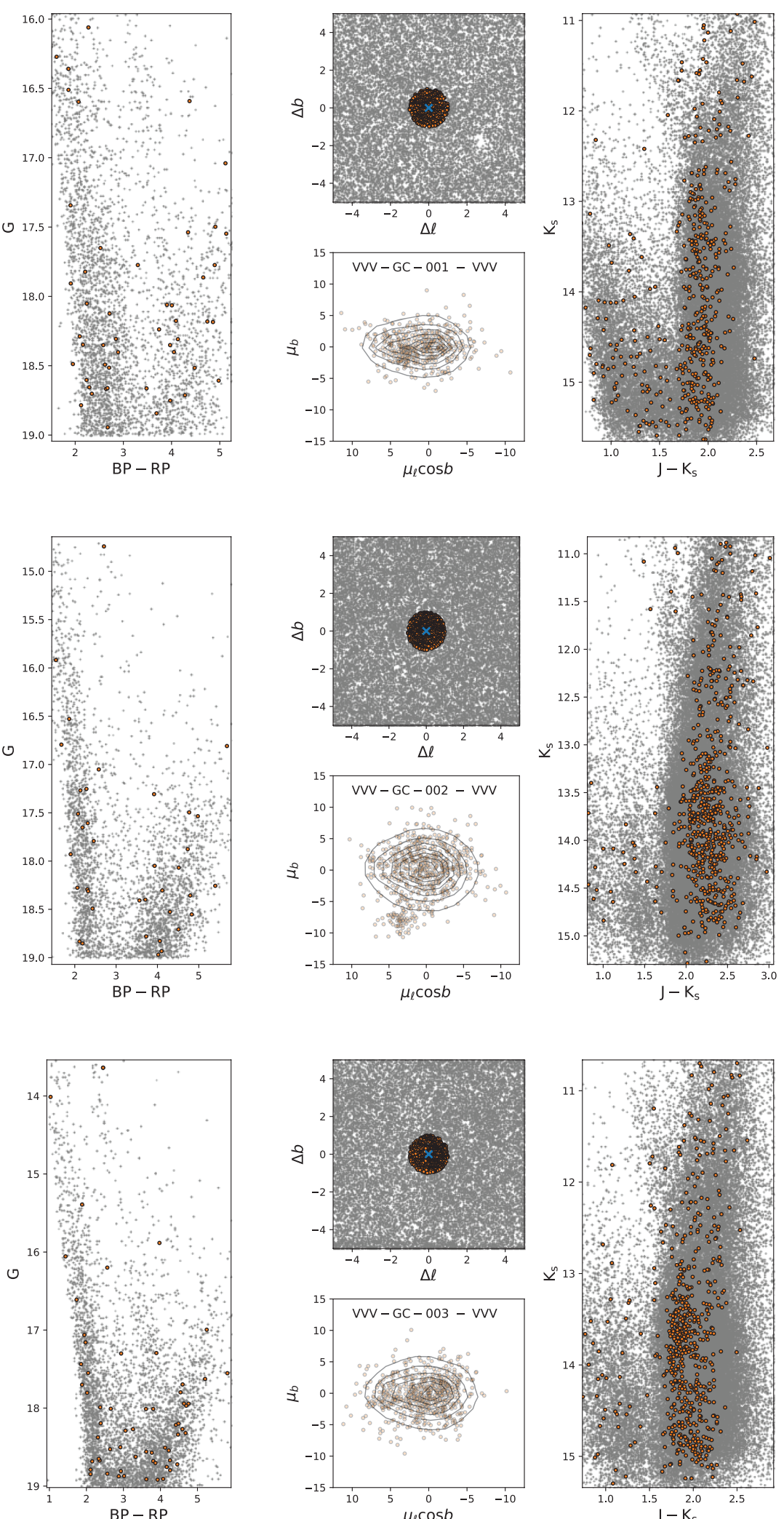
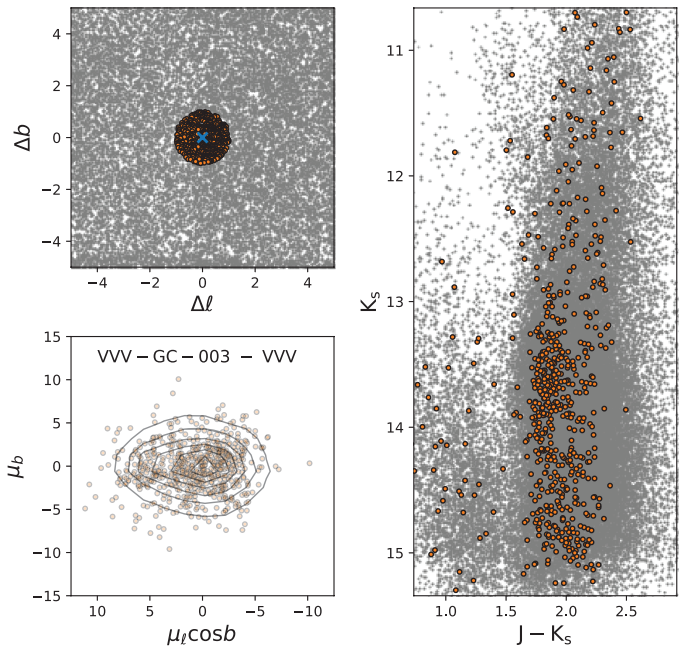

Fig. A.1. Diagnostic plots of the candidate GCs found in Gaia (left side) and VVV (right side). Each panel contains the sky position (upper left), VPD (lower left), and CMD (right) for the inner 1 arcmin (highlighted in orange) and the field stars within 10 arcmins from the nominal cluster center up to $G=19 \mathrm{mag}$. All the 93 candidate clusters but one (Minni 02) were located in the Gaia DR2 catalog and 53 were located in our VVV catalog. We recall that PMs are available from VVV only within latitudes $|b|<3^{\circ}$, plus a few fields where they were calculated specifically for other projects. 

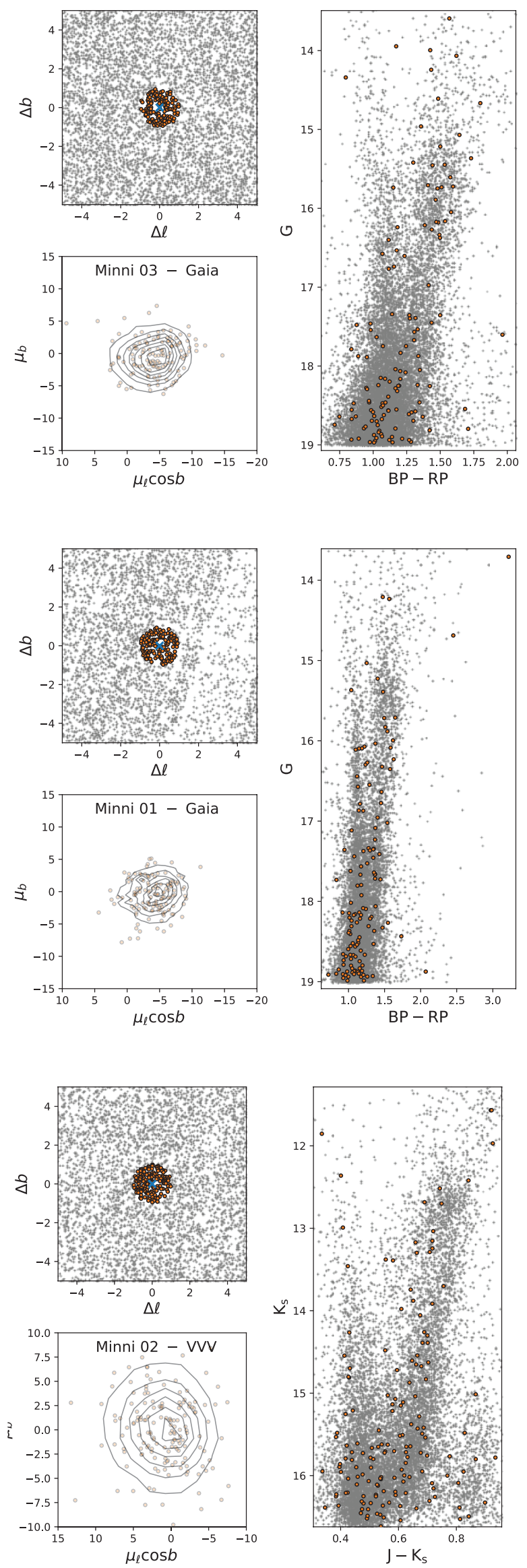
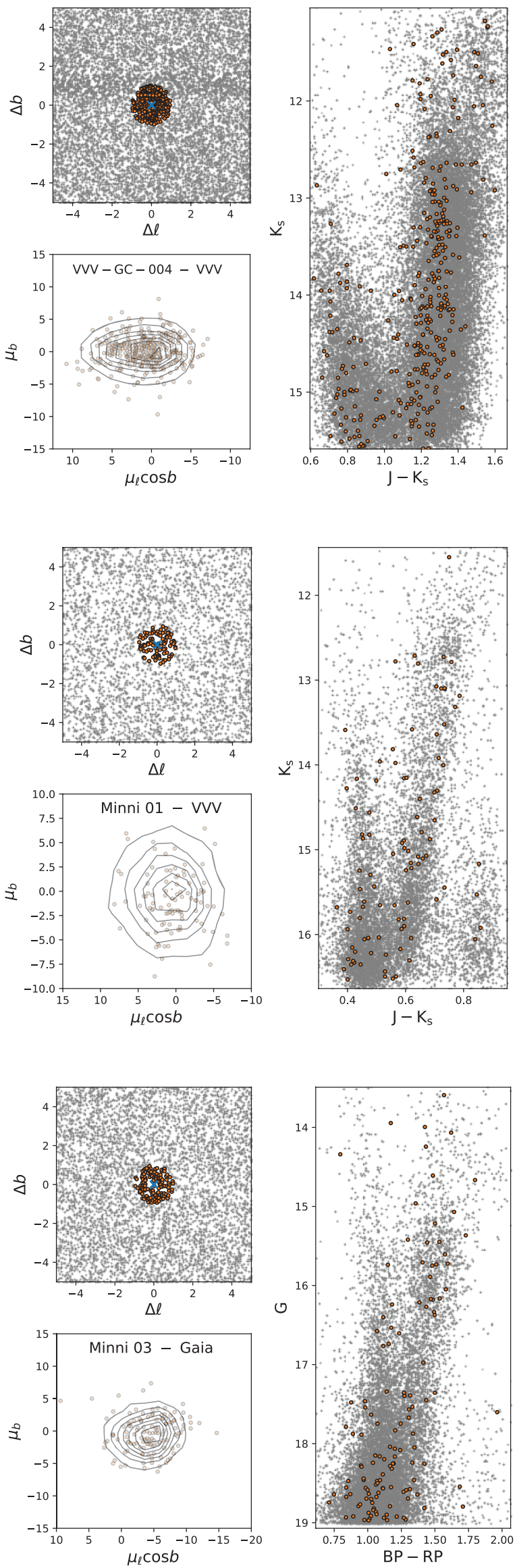

Fig. A.1. continued. 
F. Gran et al.: Gaia and VVV view of the last bulge globular cluster candidates
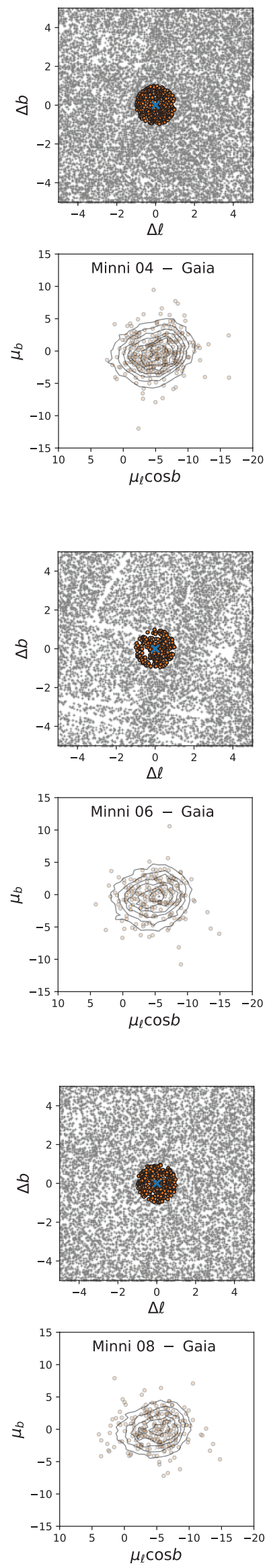
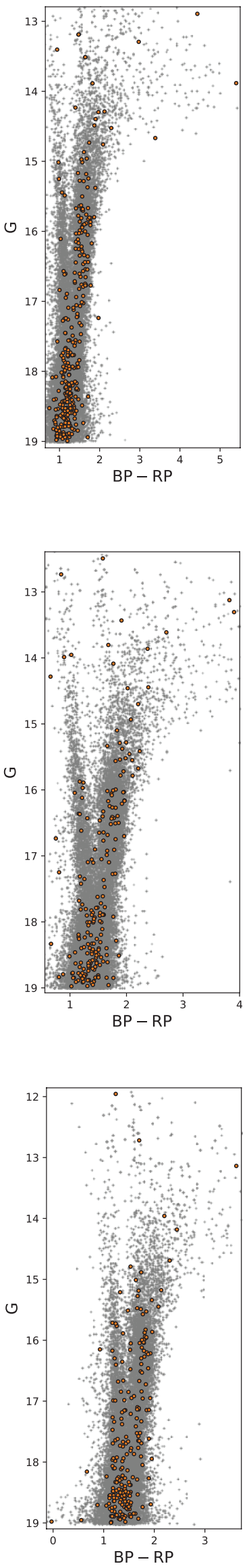
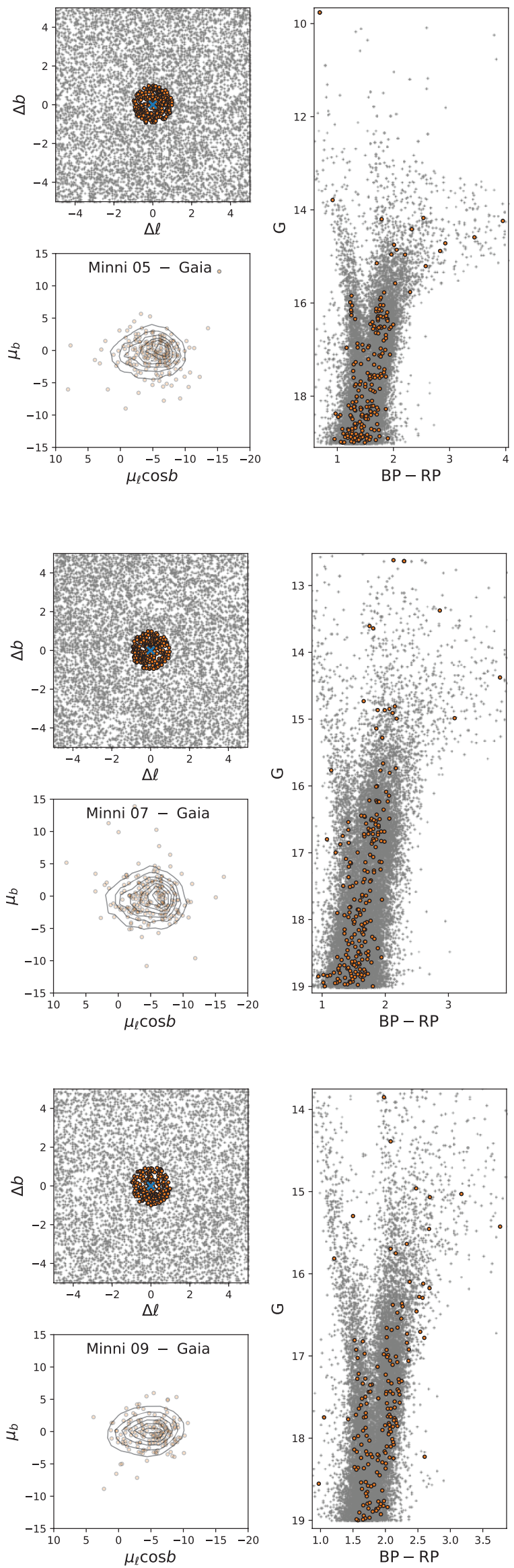

Fig. A.1. continued. 

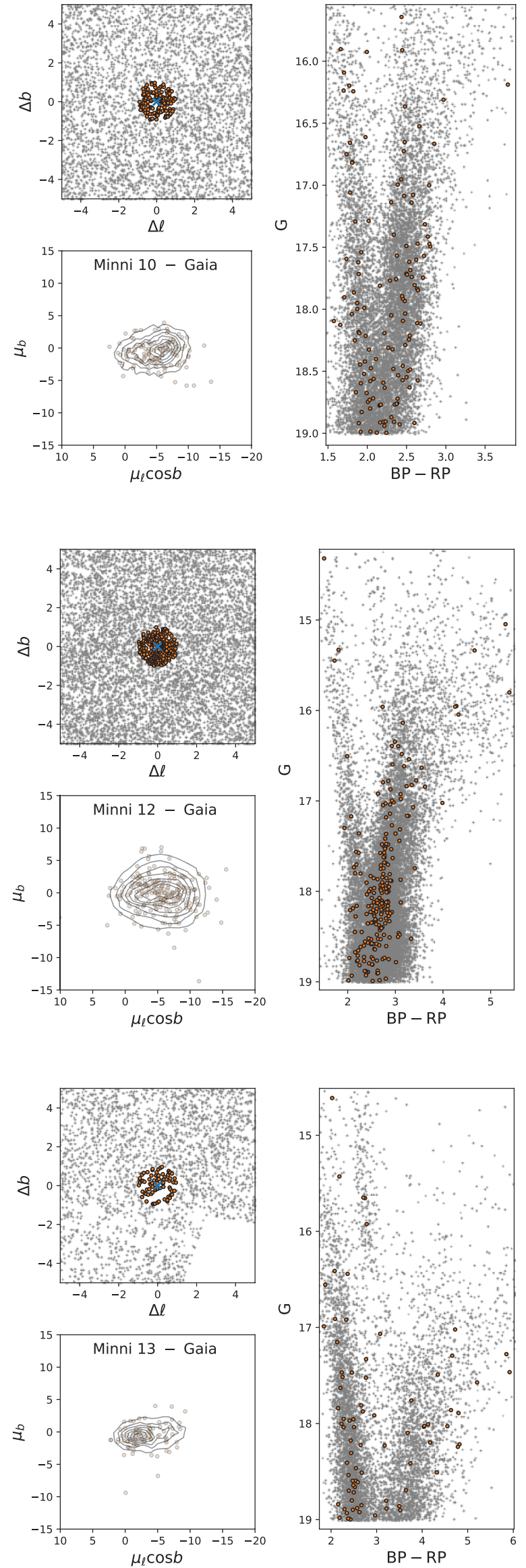
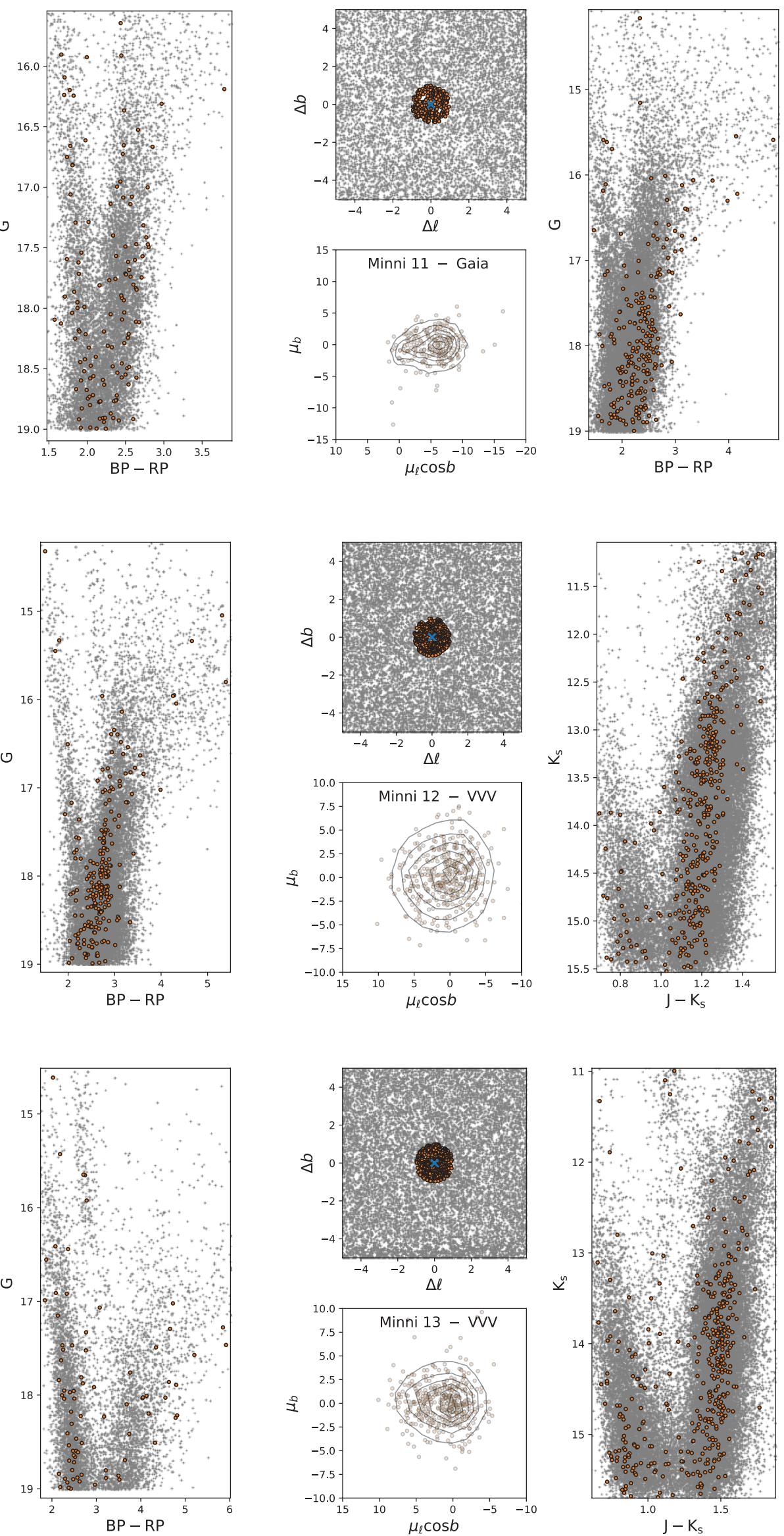

Fig. A.1. continued. 
F. Gran et al.: Gaia and VVV view of the last bulge globular cluster candidates
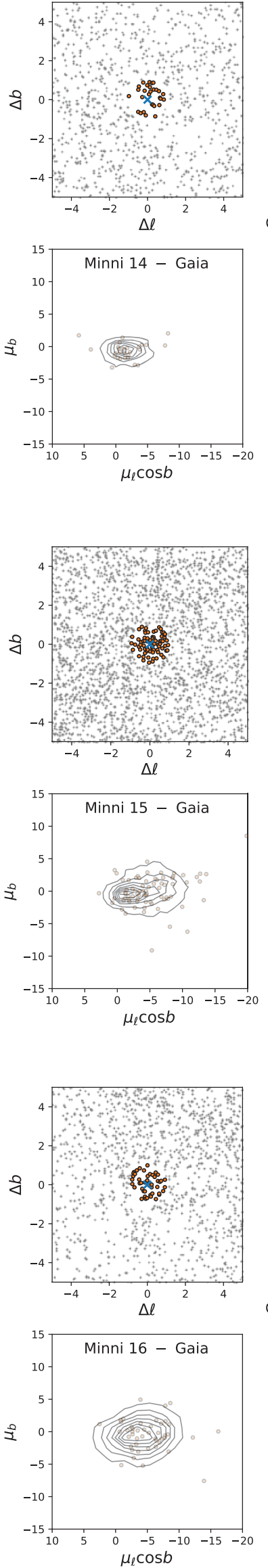
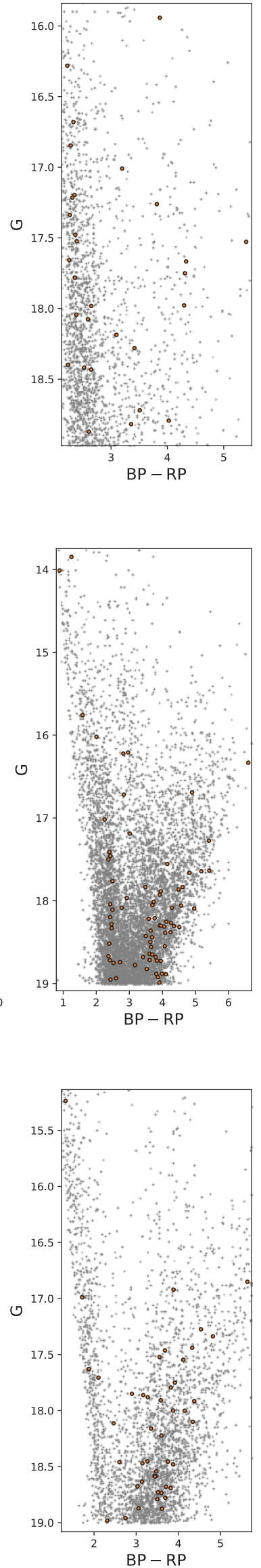
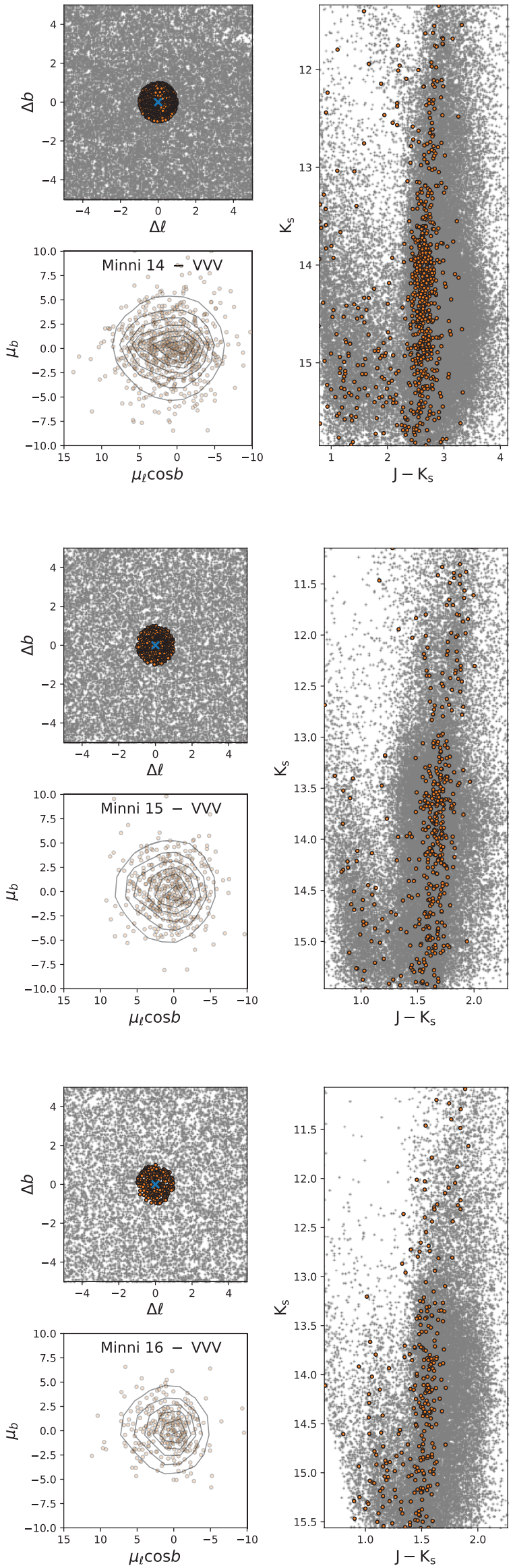

Fig. A.1. continued. 

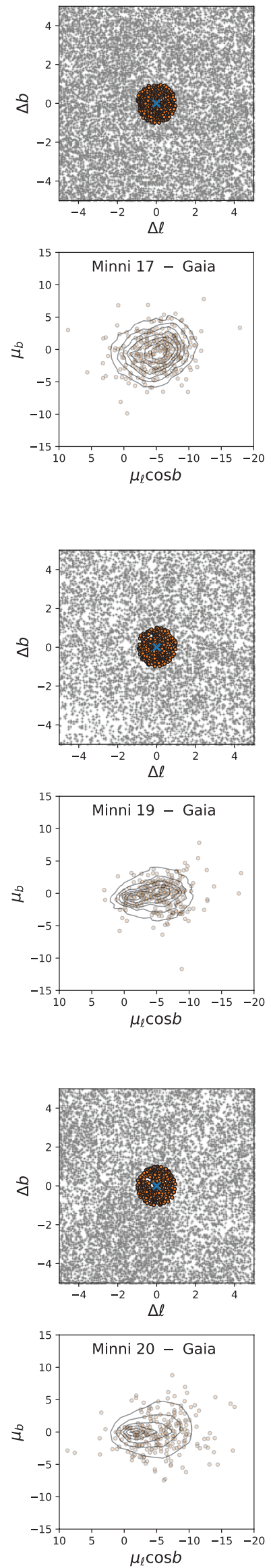
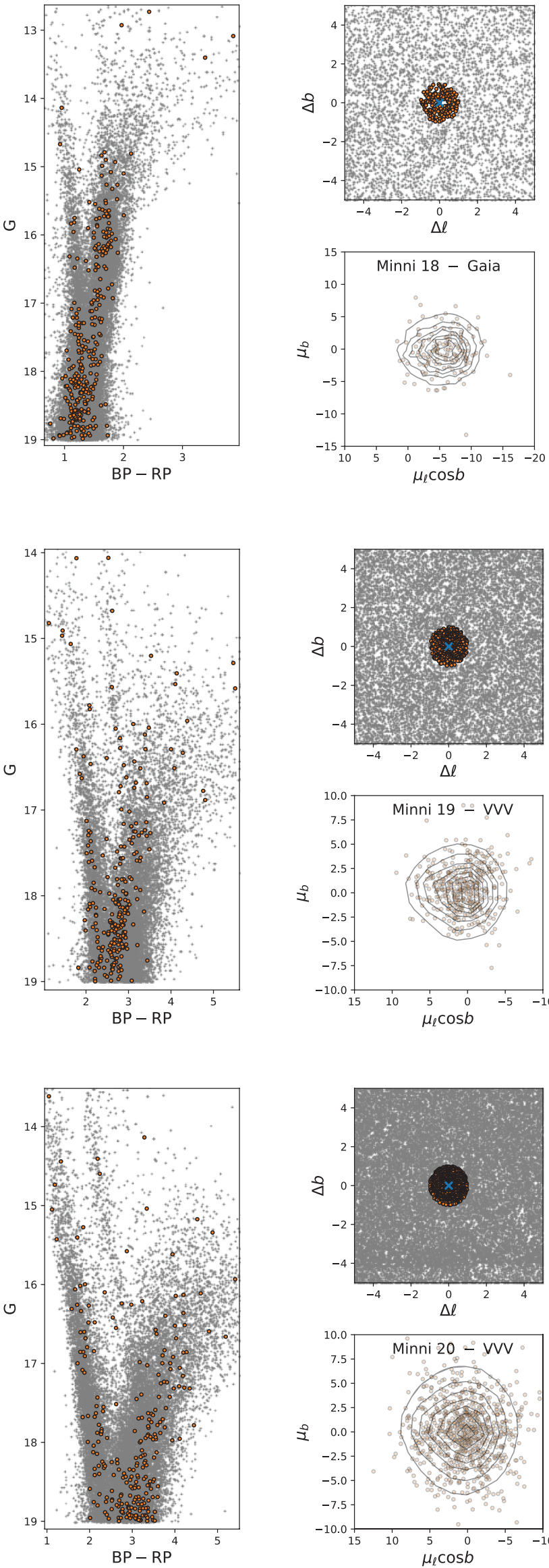
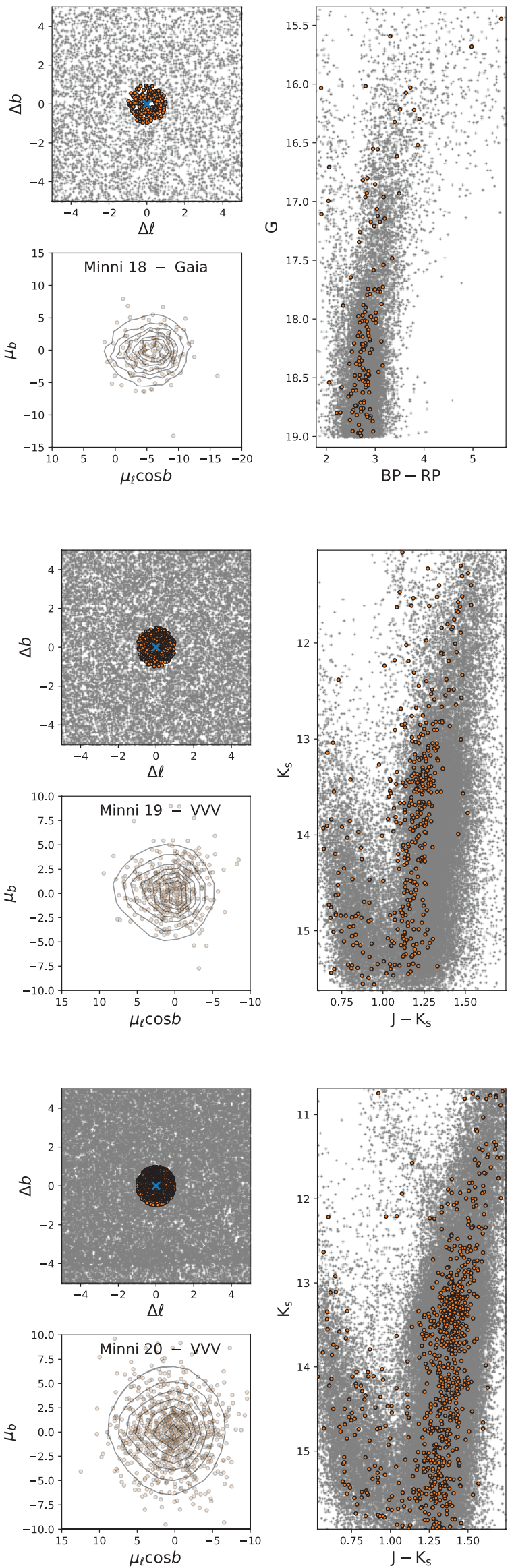

Fig. A.1. continued. 
F. Gran et al.: Gaia and VVV view of the last bulge globular cluster candidates
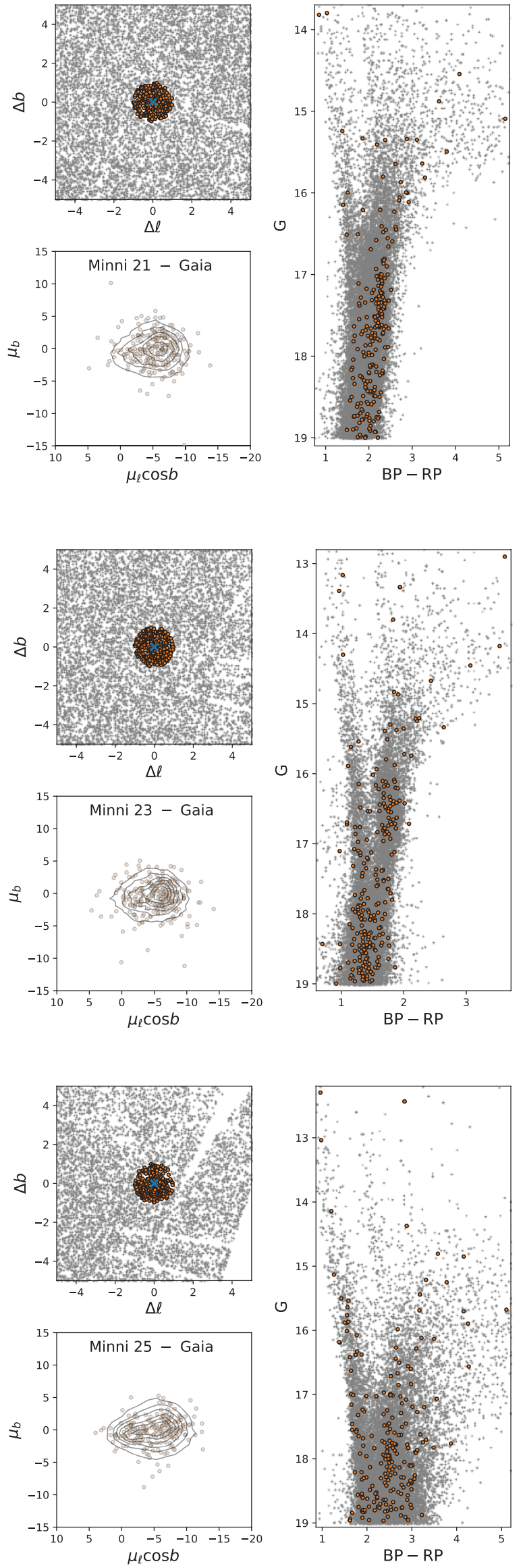
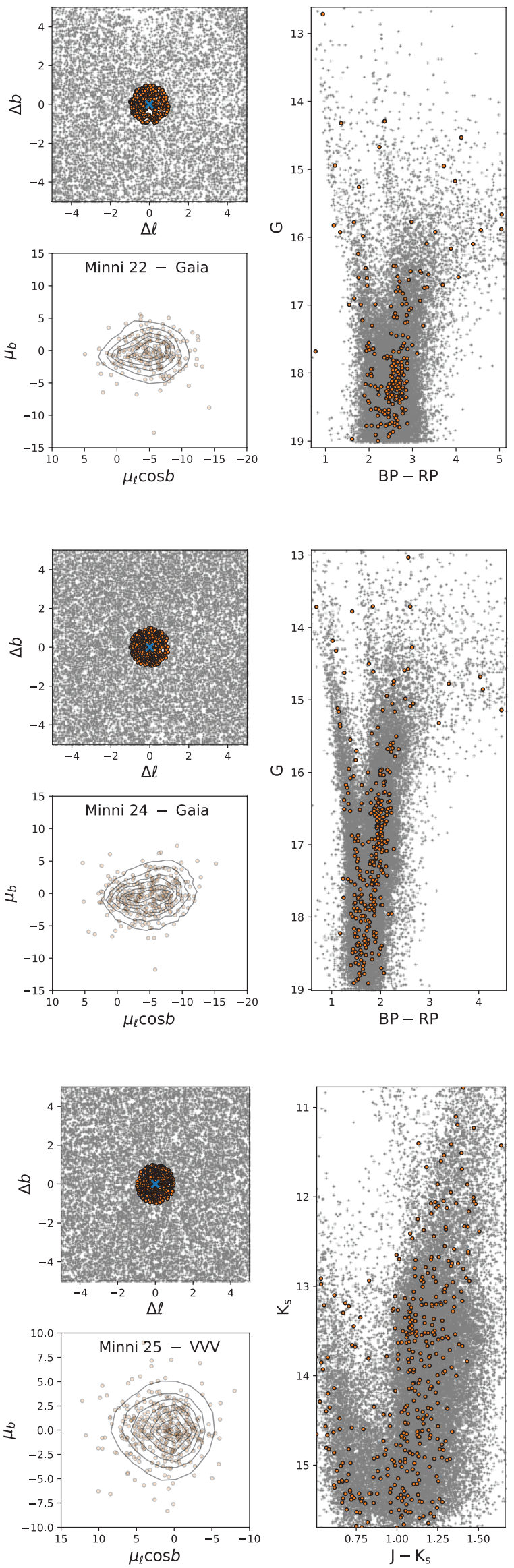

Fig. A.1. continued. 

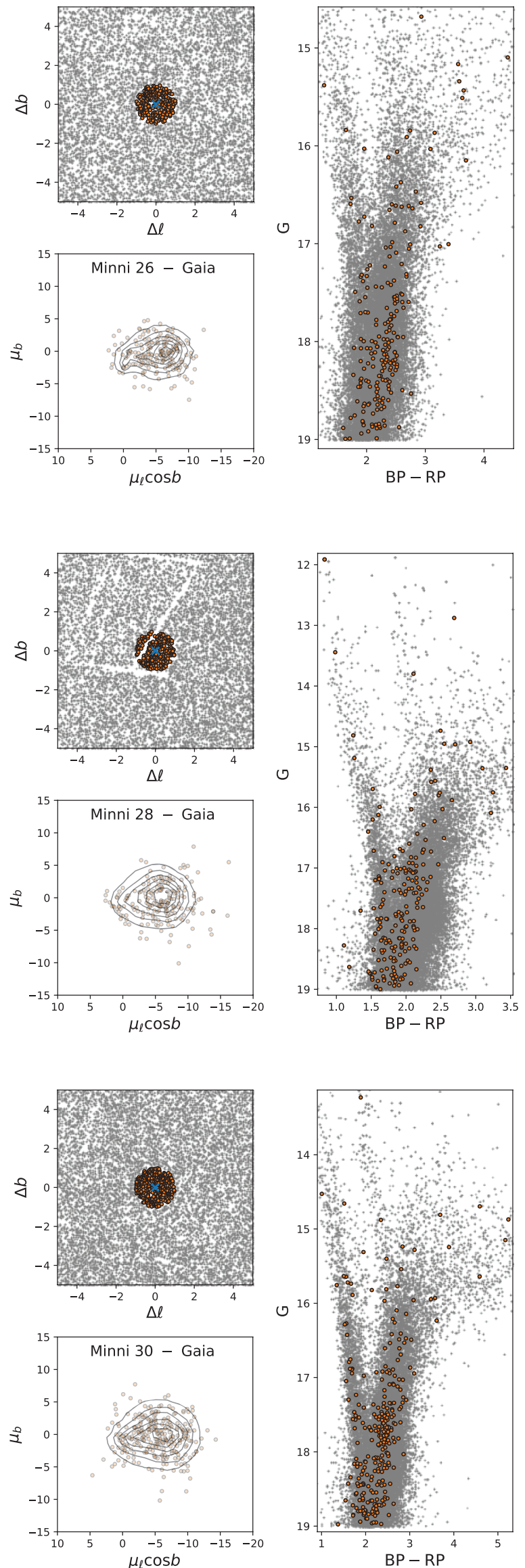
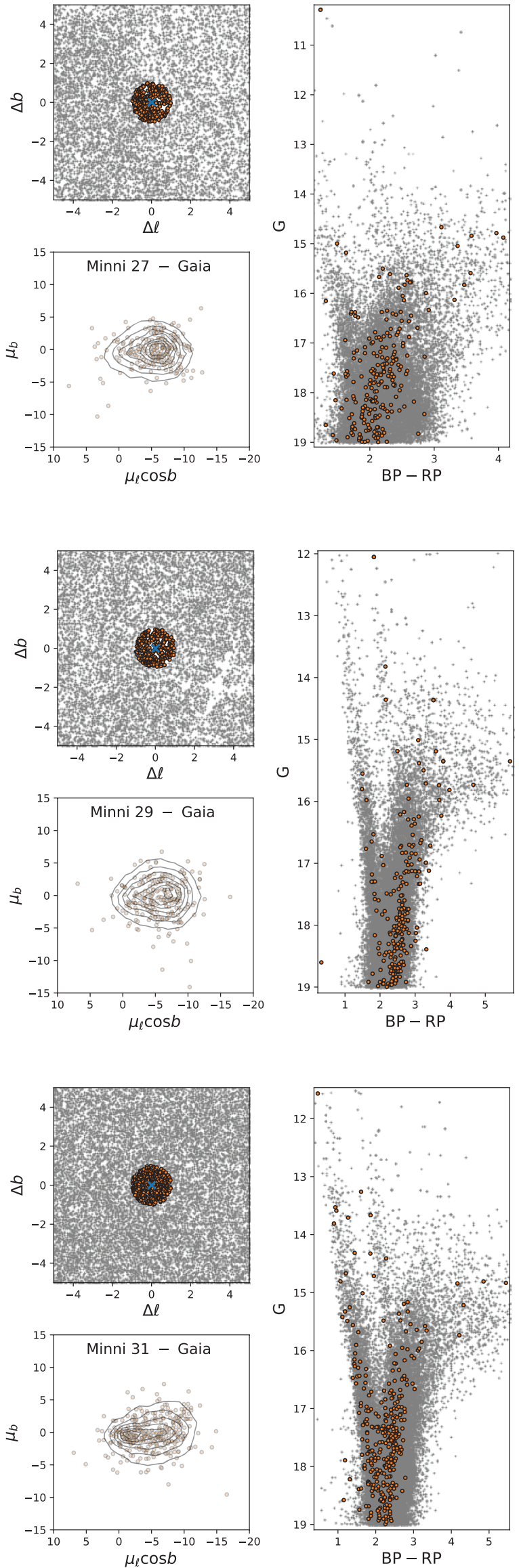

Fig. A.1. continued. 
F. Gran et al.: Gaia and VVV view of the last bulge globular cluster candidates
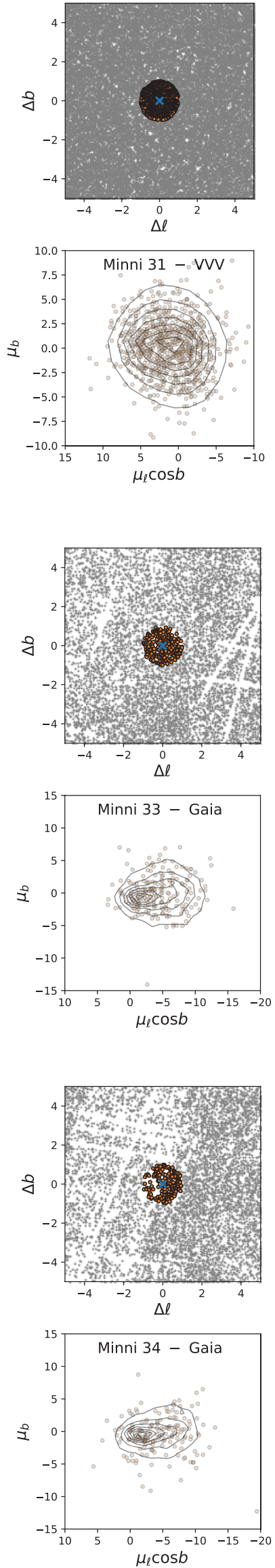
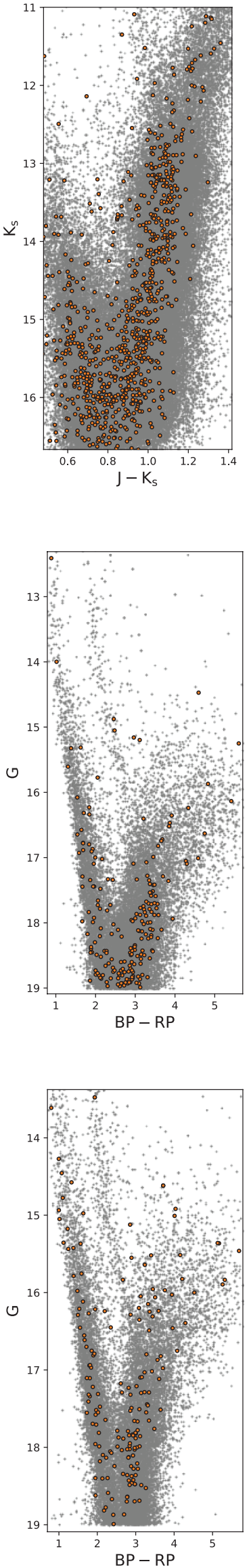
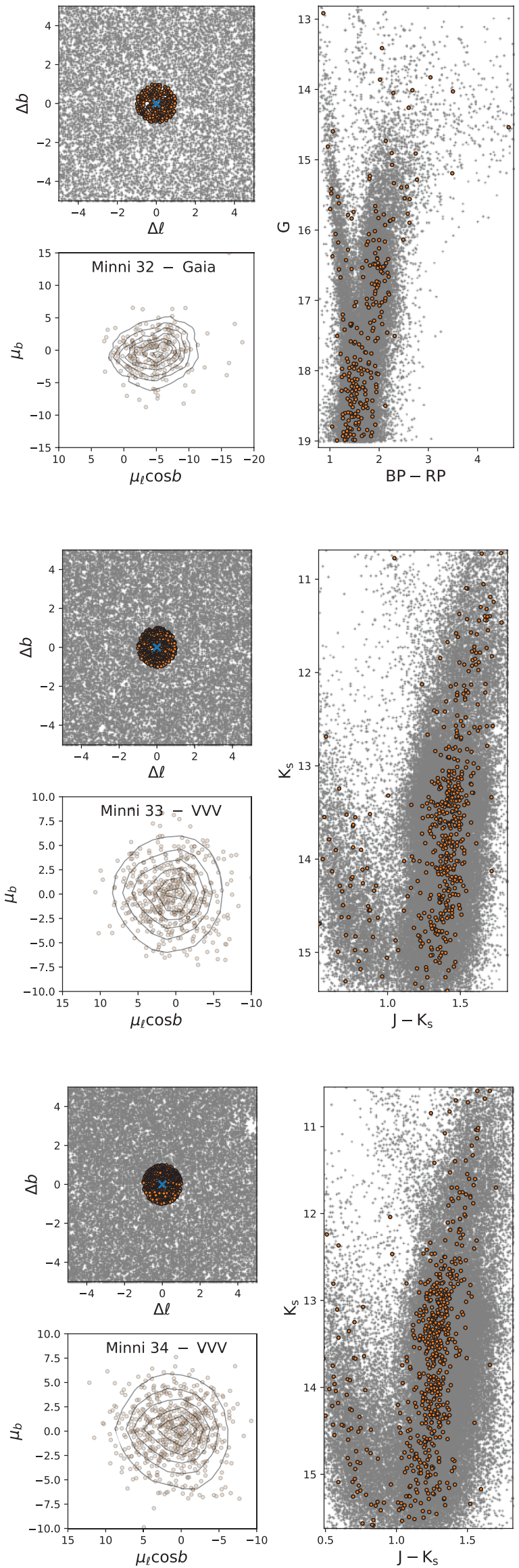

Fig. A.1. continued. 

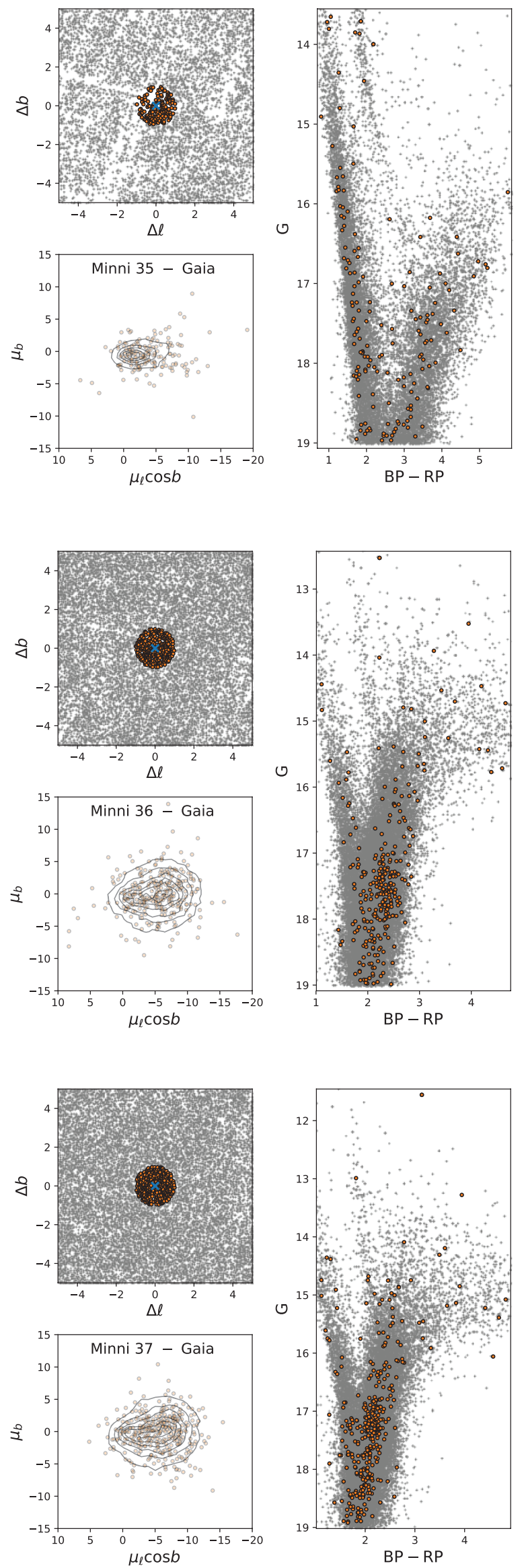
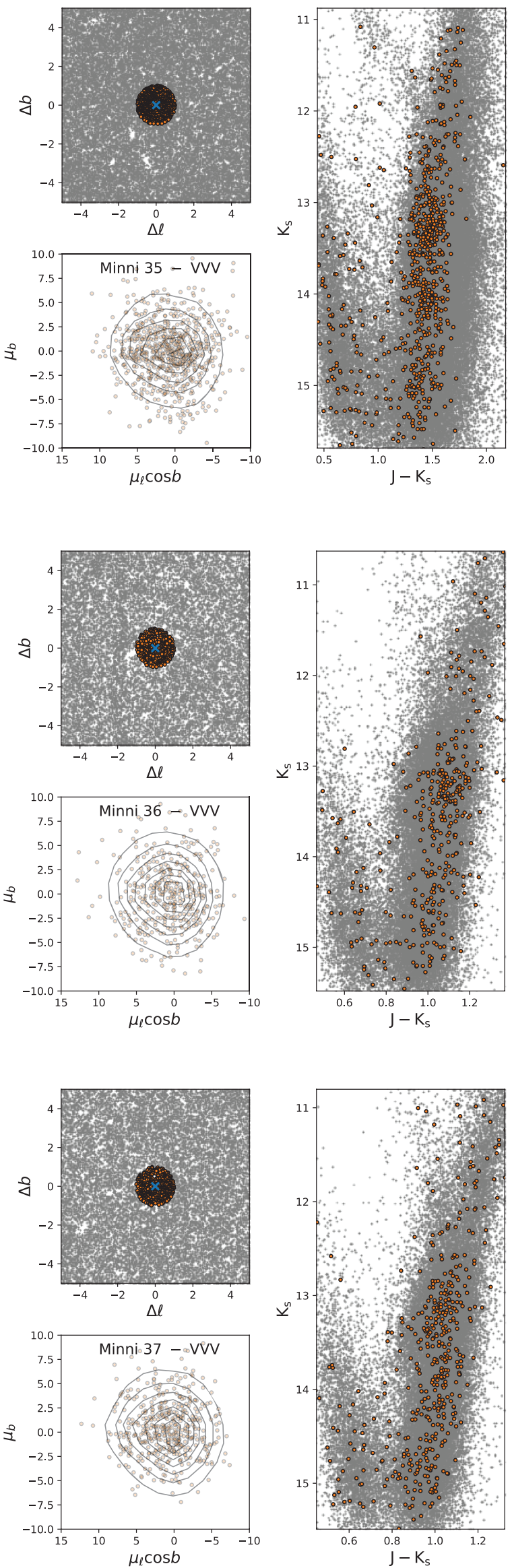

Fig. A.1. continued. 
F. Gran et al.: Gaia and VVV view of the last bulge globular cluster candidates
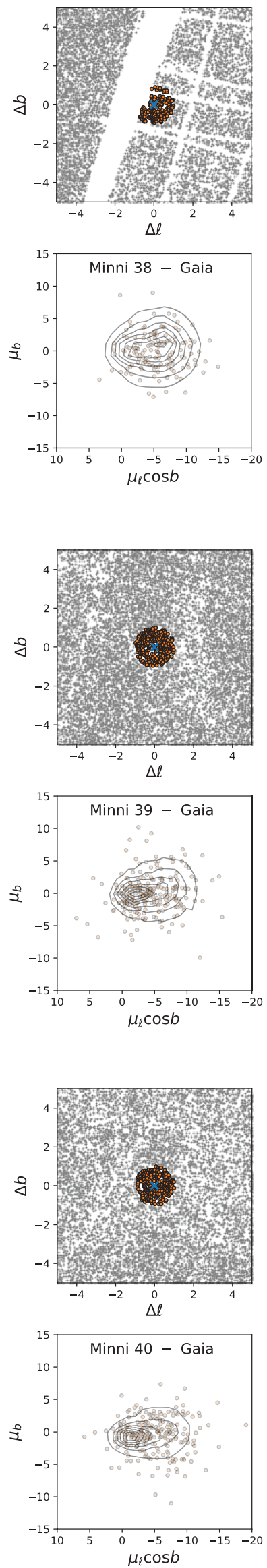
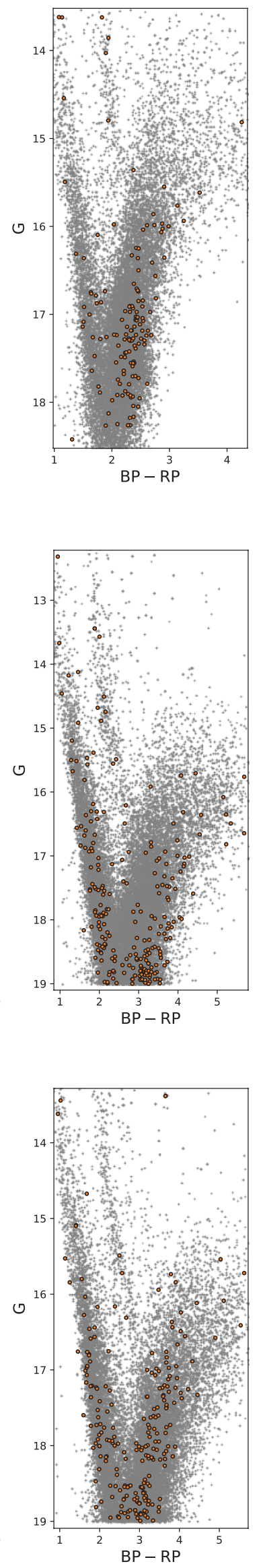
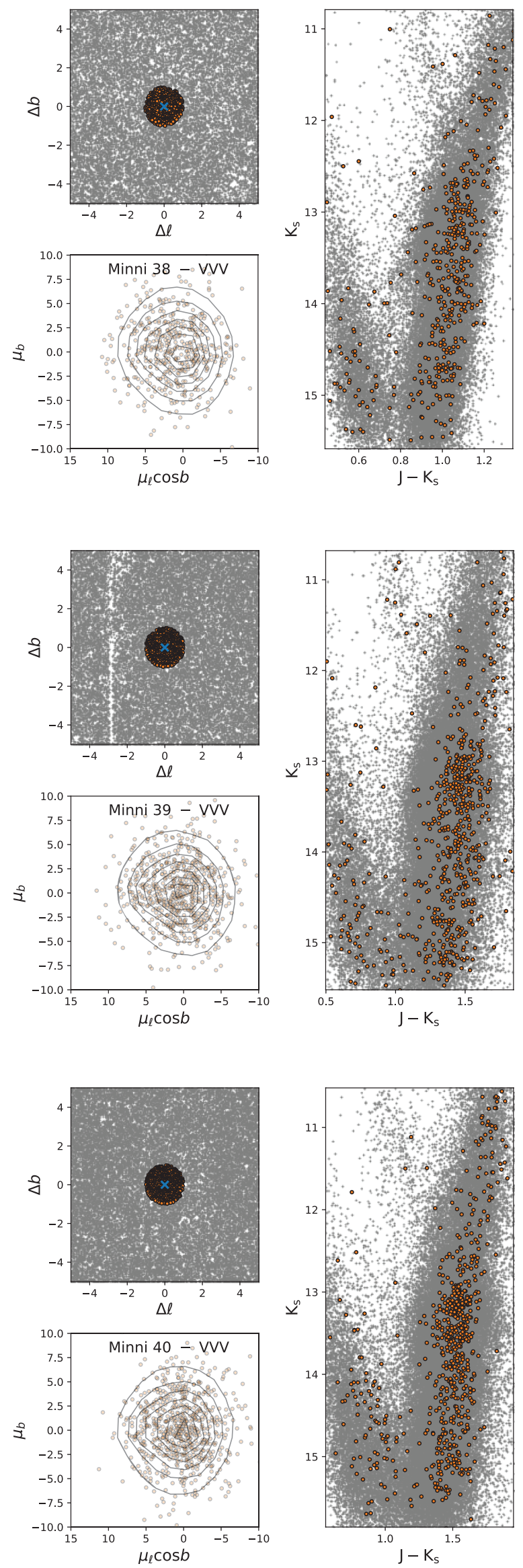

Fig. A.1. continued. 
A\&A 628, A45 (2019)
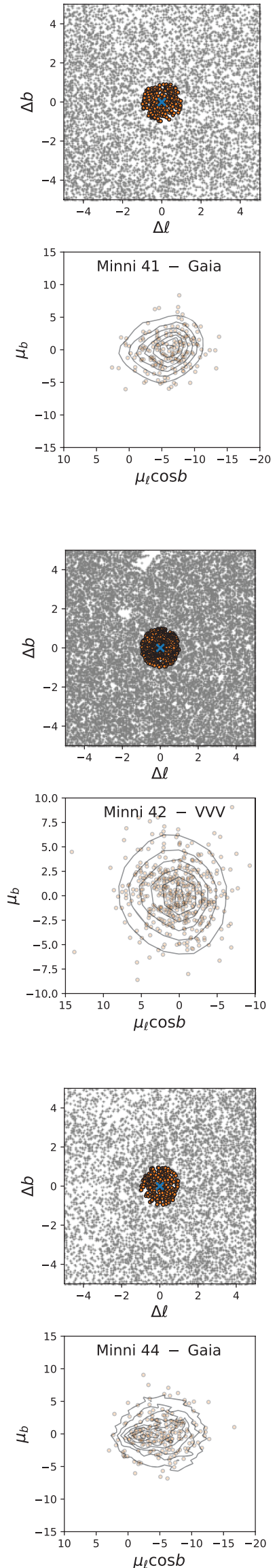
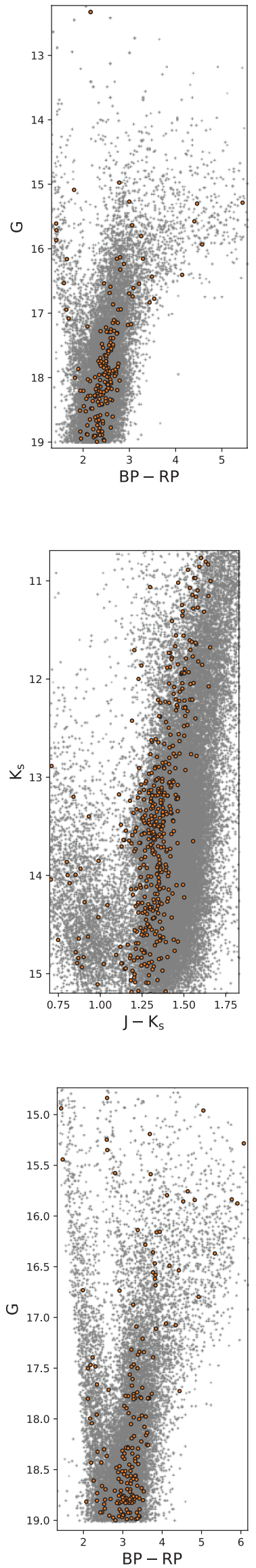
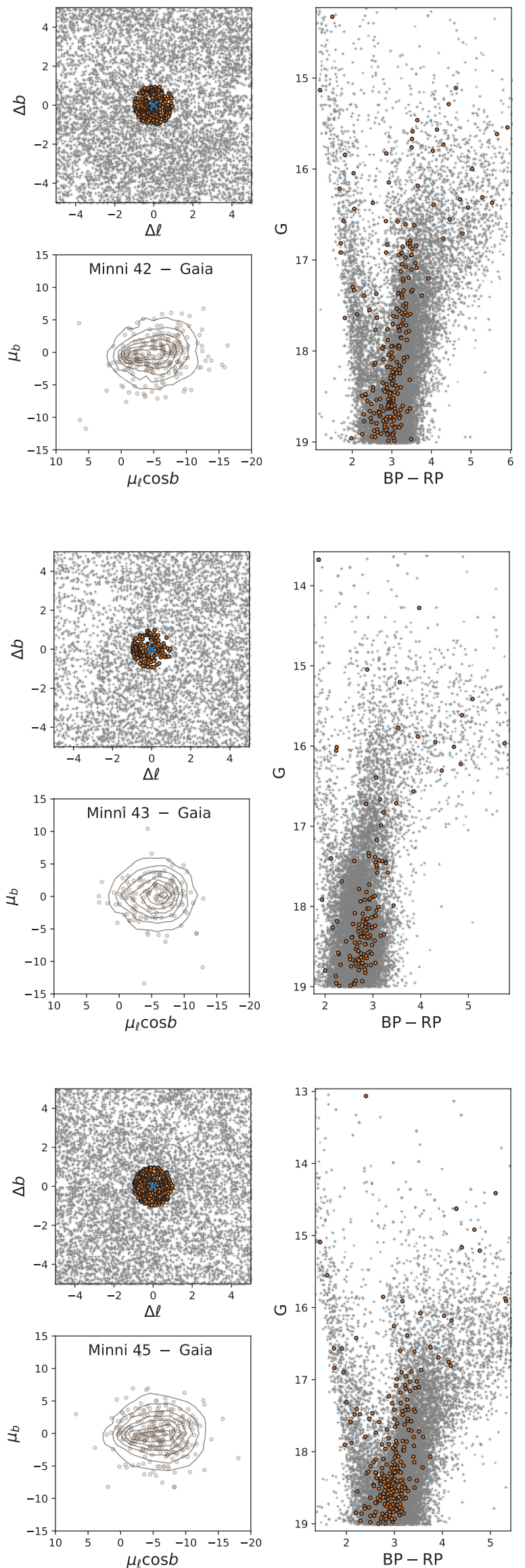

Fig. A.1. continued. 
F. Gran et al.: Gaia and VVV view of the last bulge globular cluster candidates
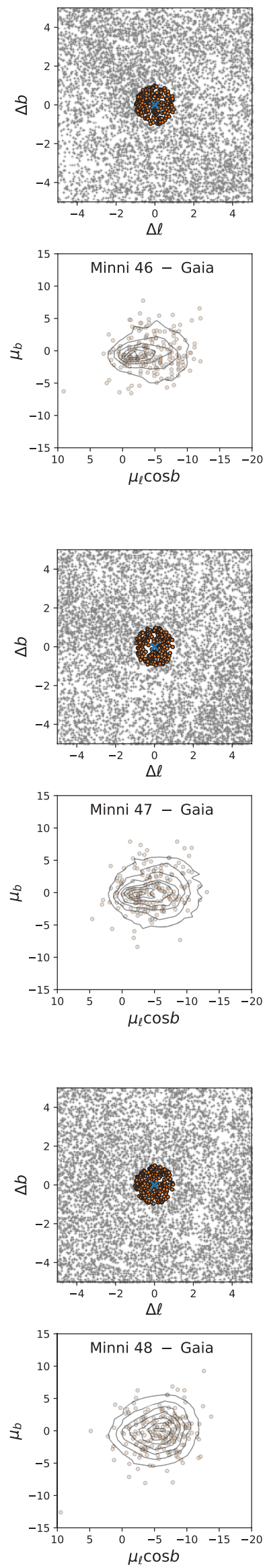
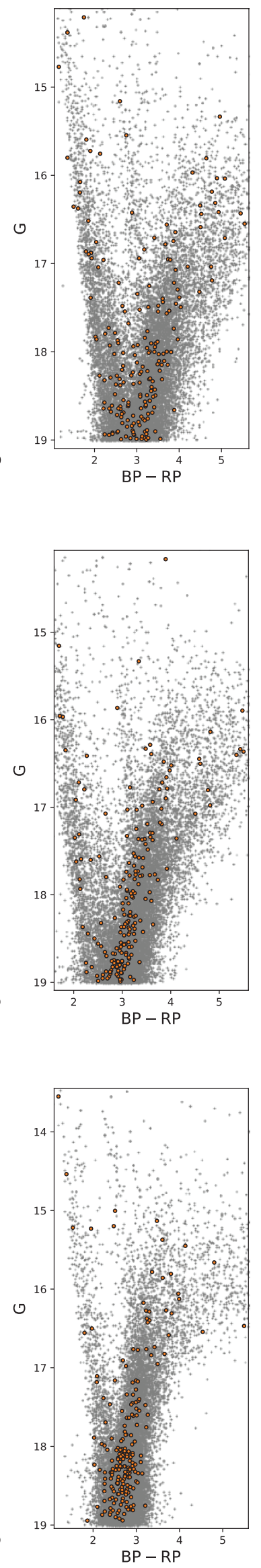
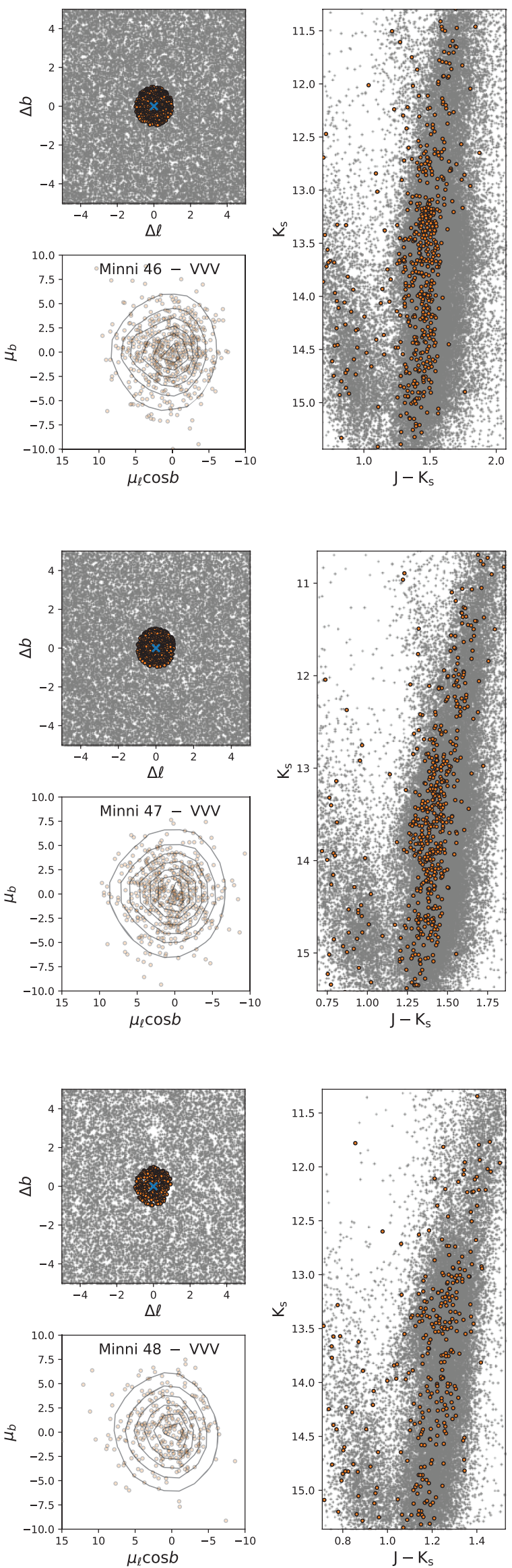

Fig. A.1. continued. 

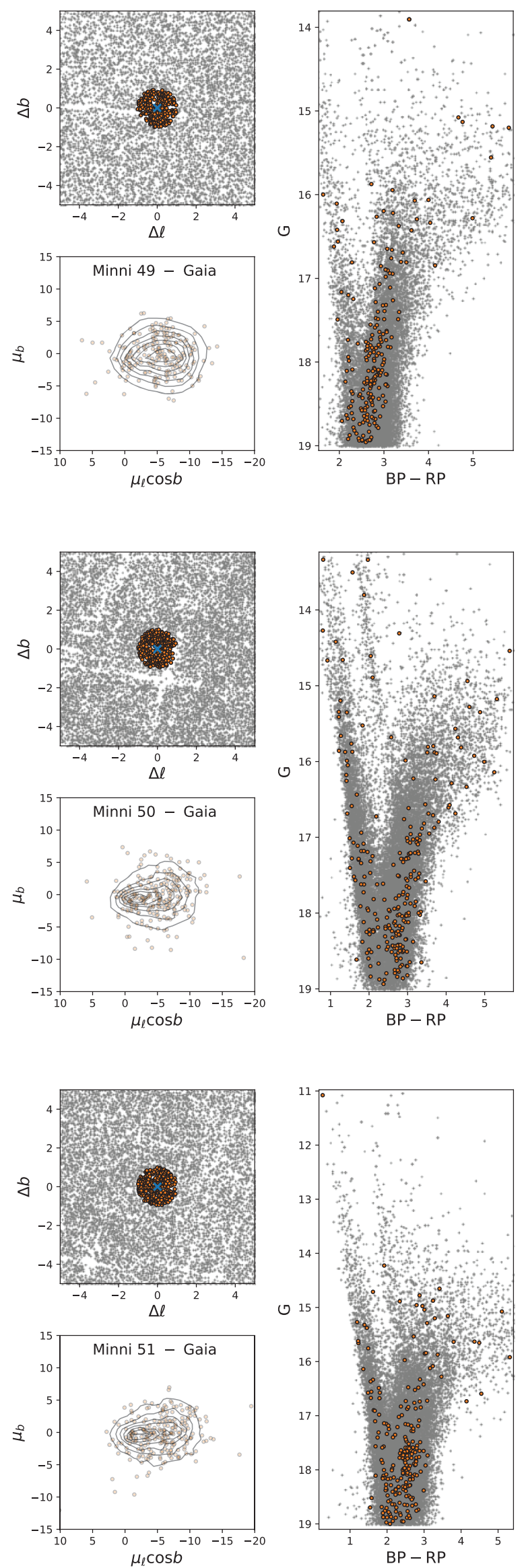
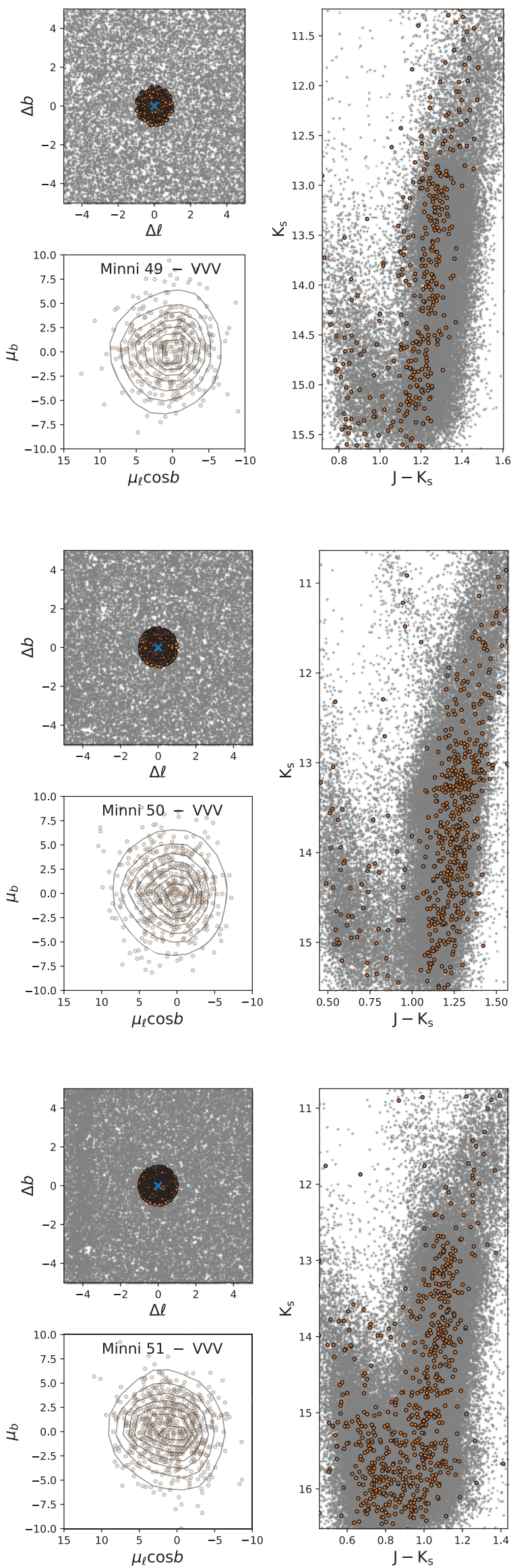

Fig. A.1. continued. 
F. Gran et al.: Gaia and VVV view of the last bulge globular cluster candidates
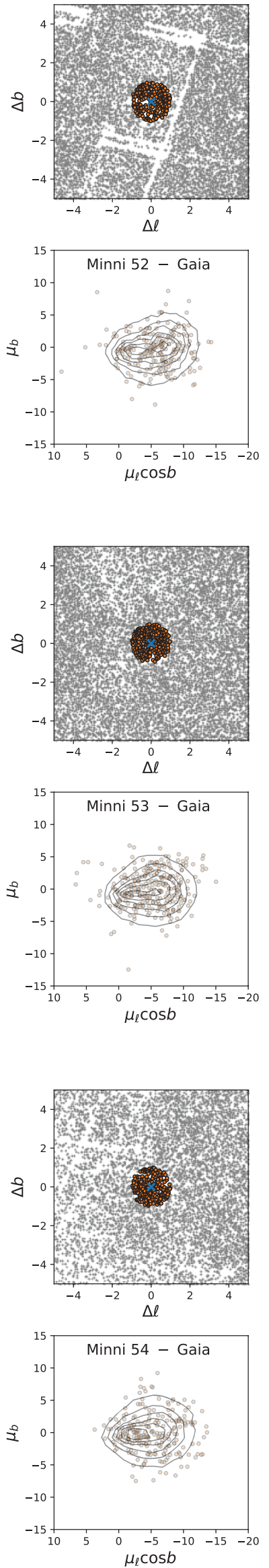
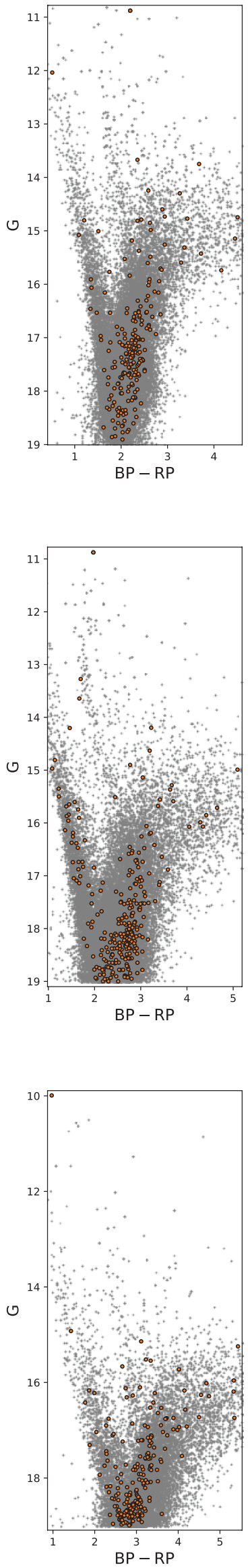
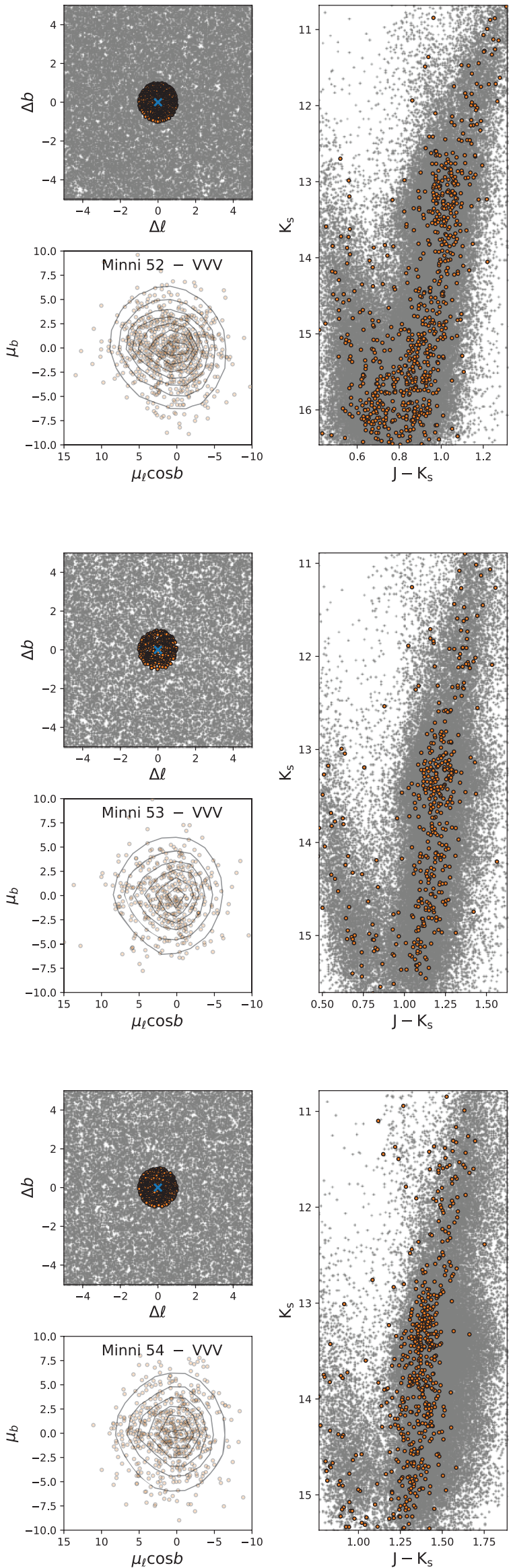

Fig. A.1. continued. 

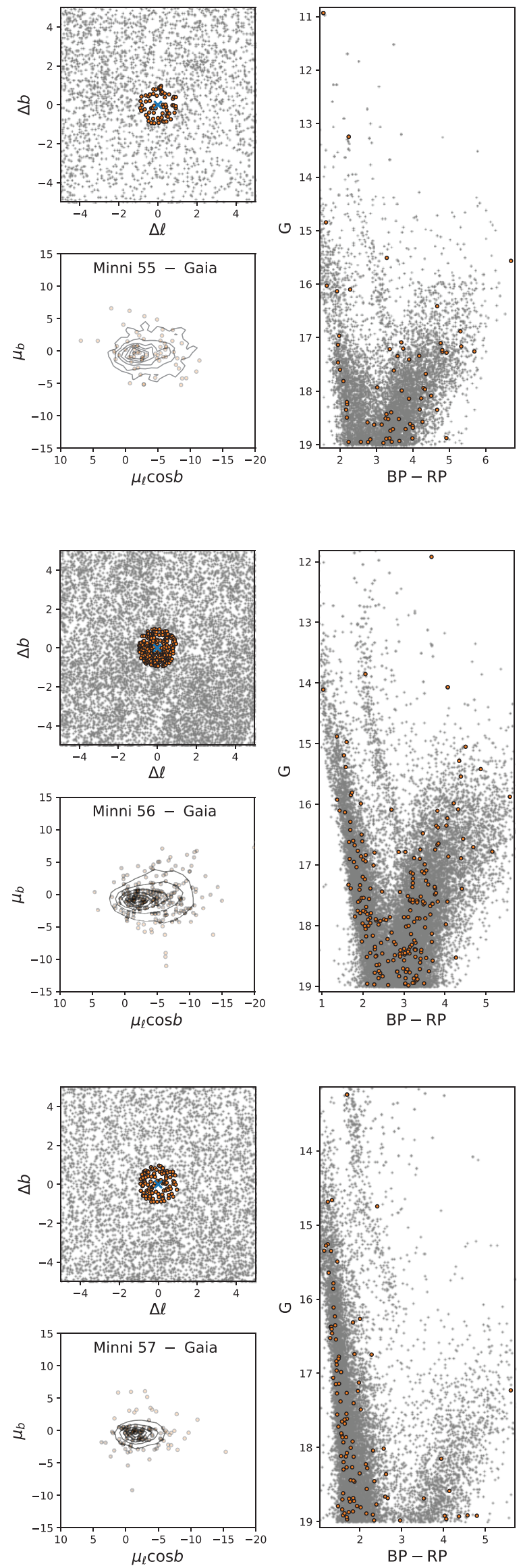
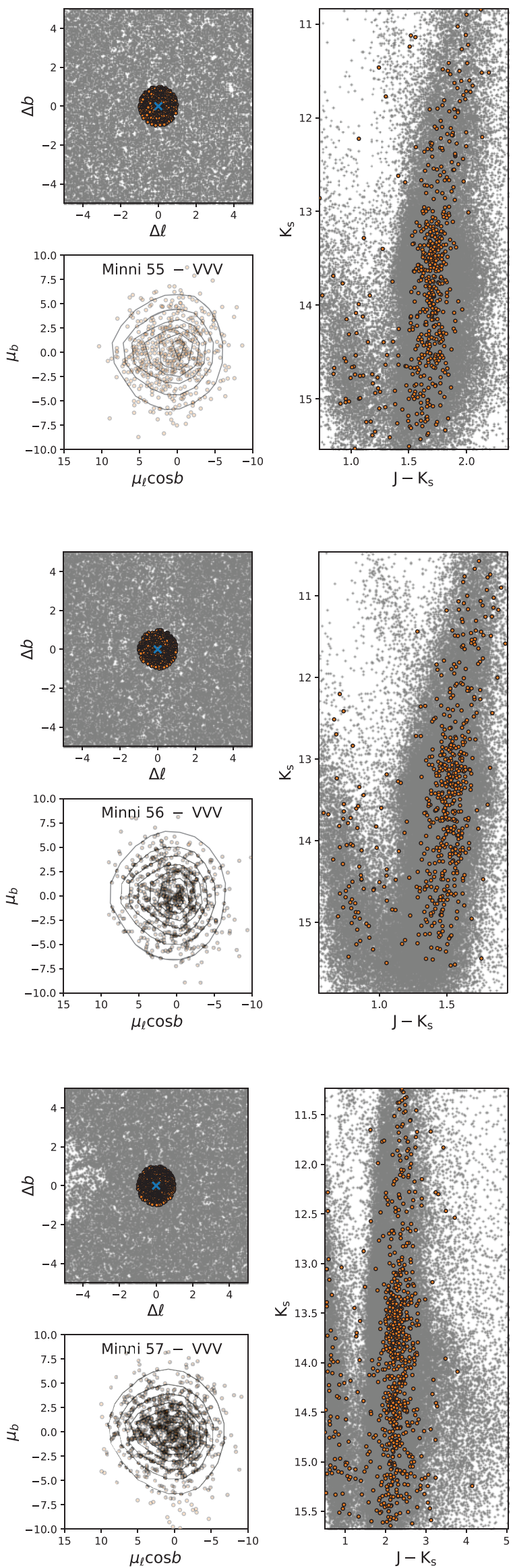

Fig. A.1. continued. 
F. Gran et al.: Gaia and VVV view of the last bulge globular cluster candidates
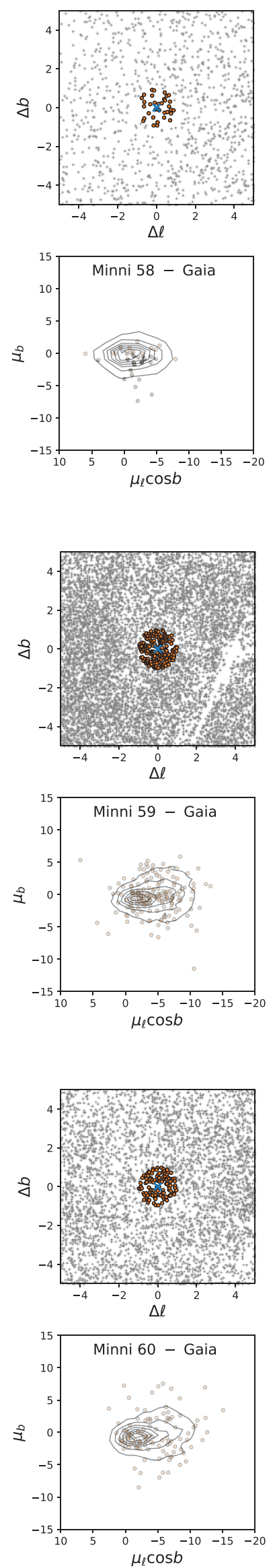
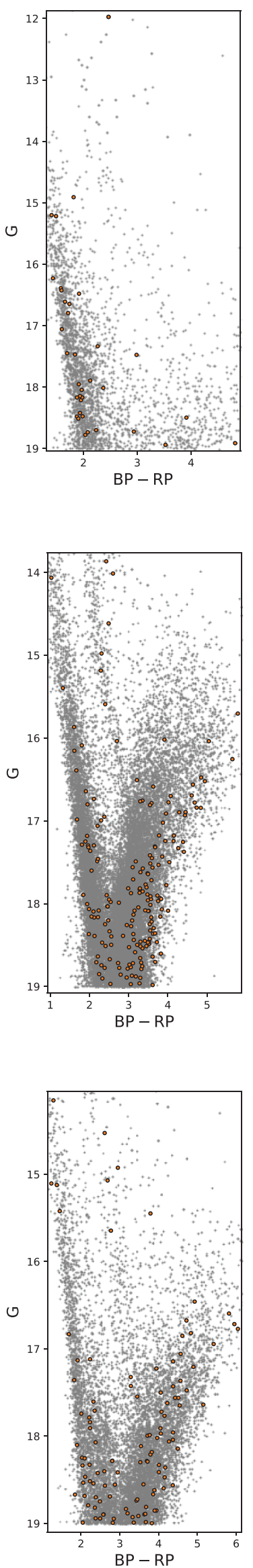
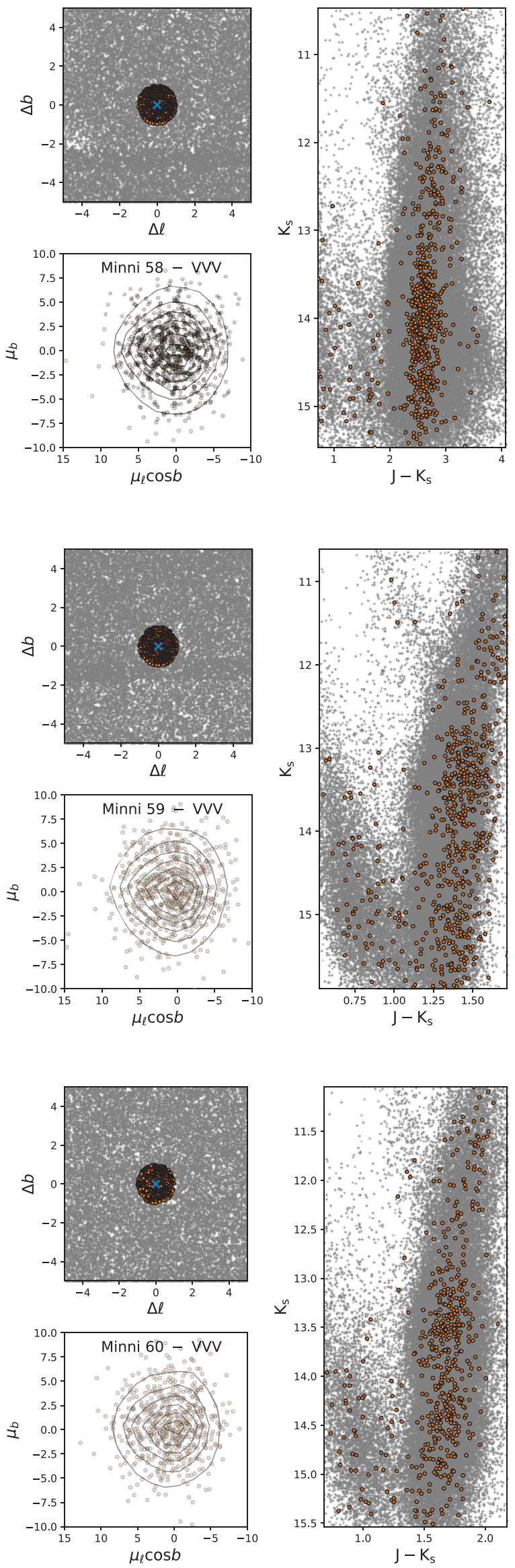

Fig. A.1. continued. 
A\&A 628, A45 (2019)
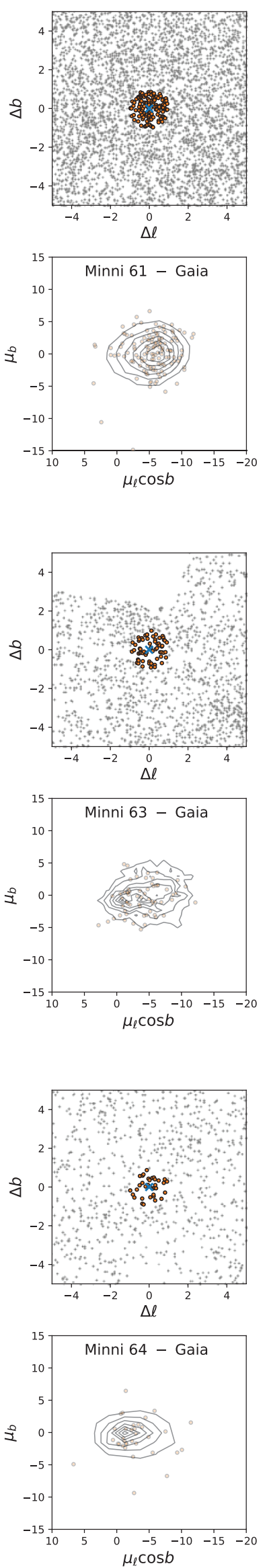
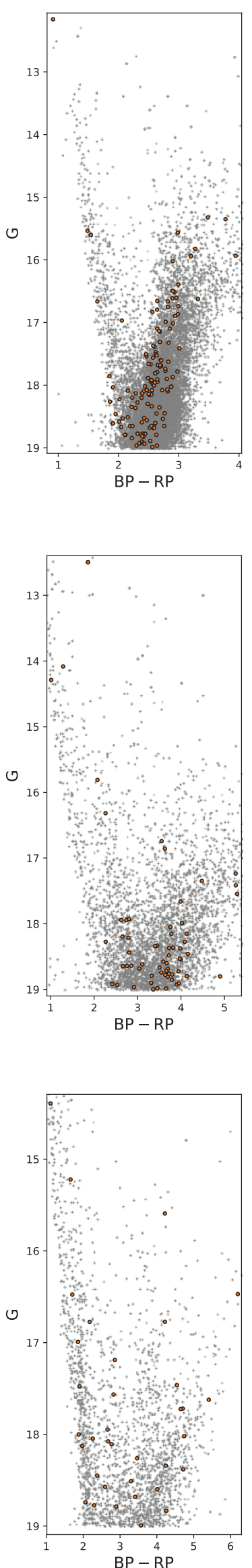
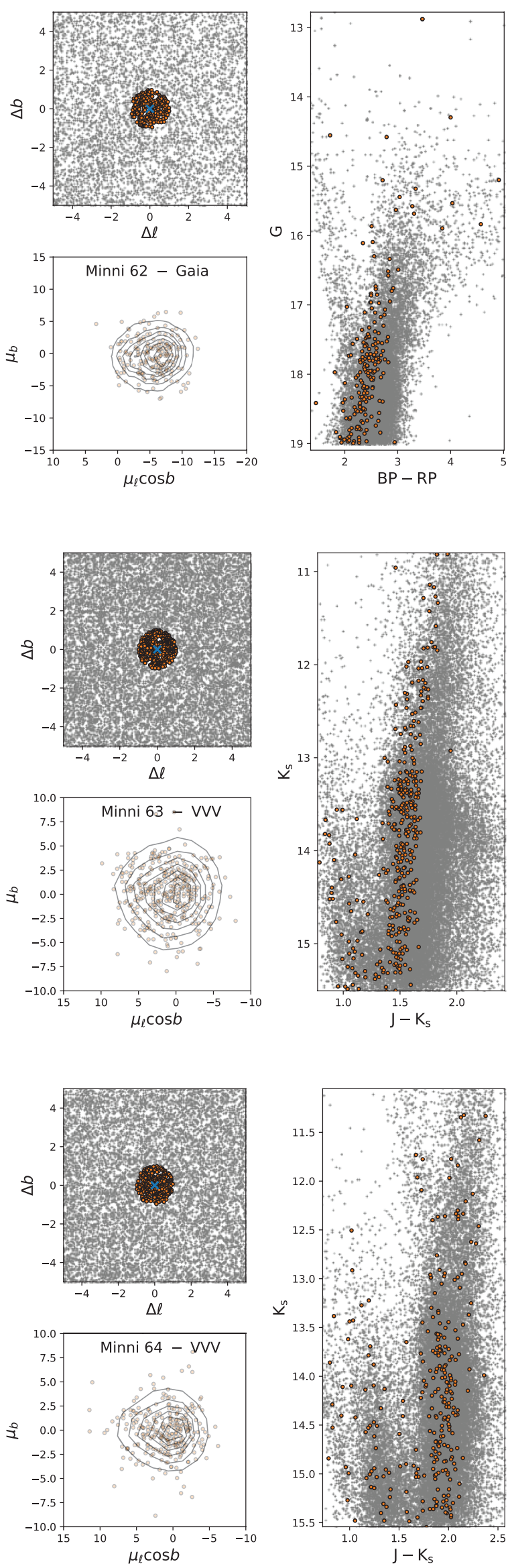

Fig. A.1. continued. 
F. Gran et al.: Gaia and VVV view of the last bulge globular cluster candidates
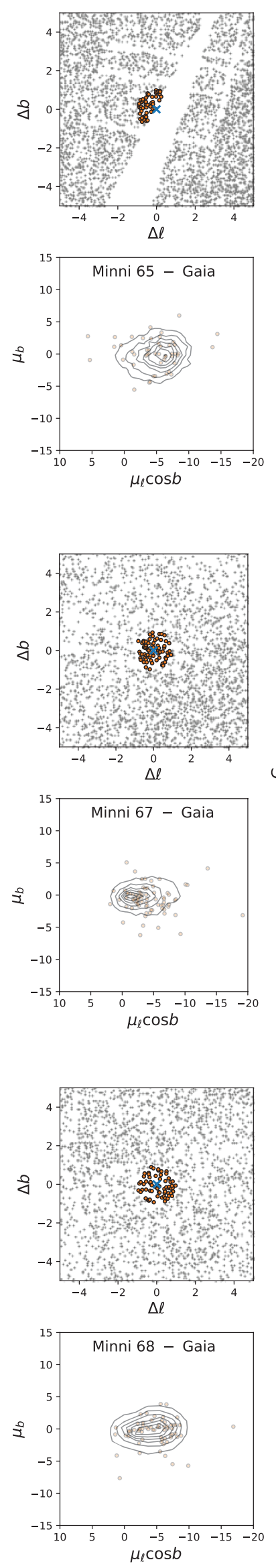

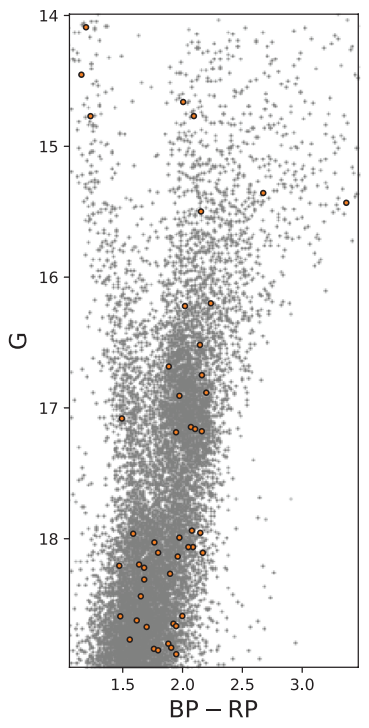

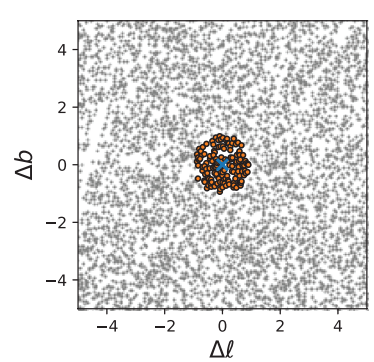
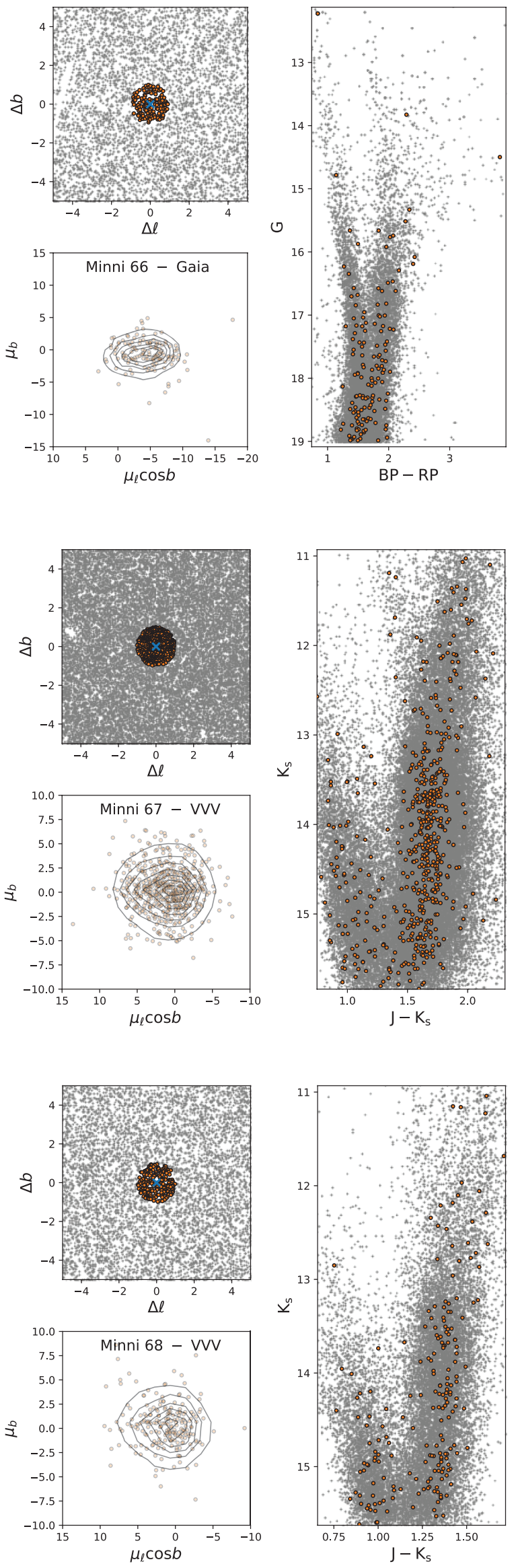

Fig. A.1. continued.

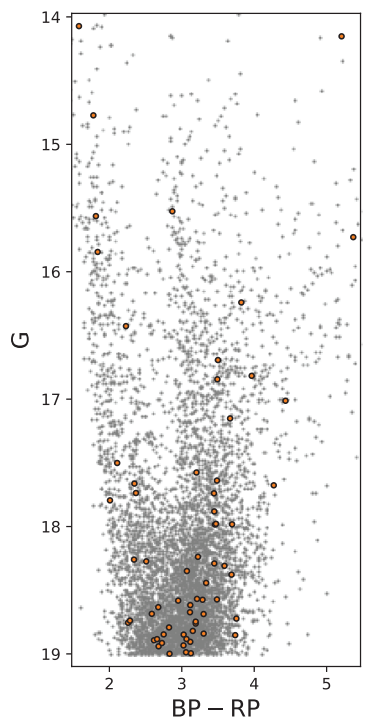

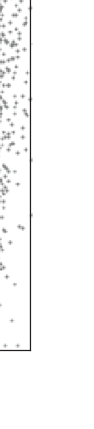



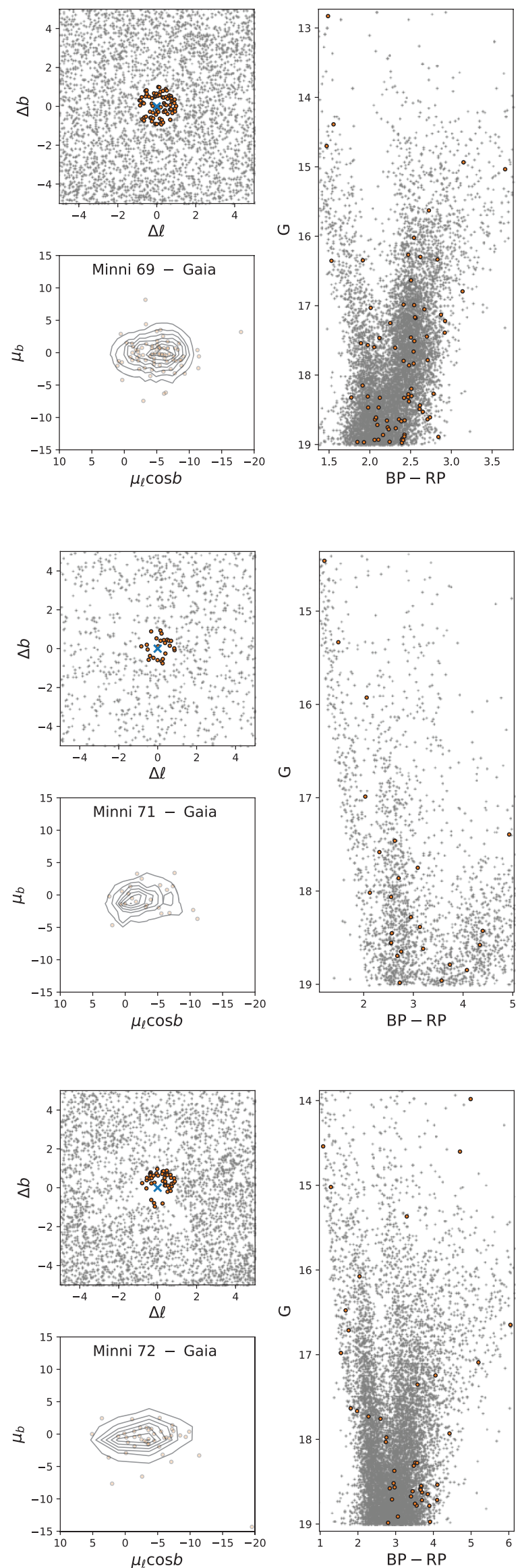
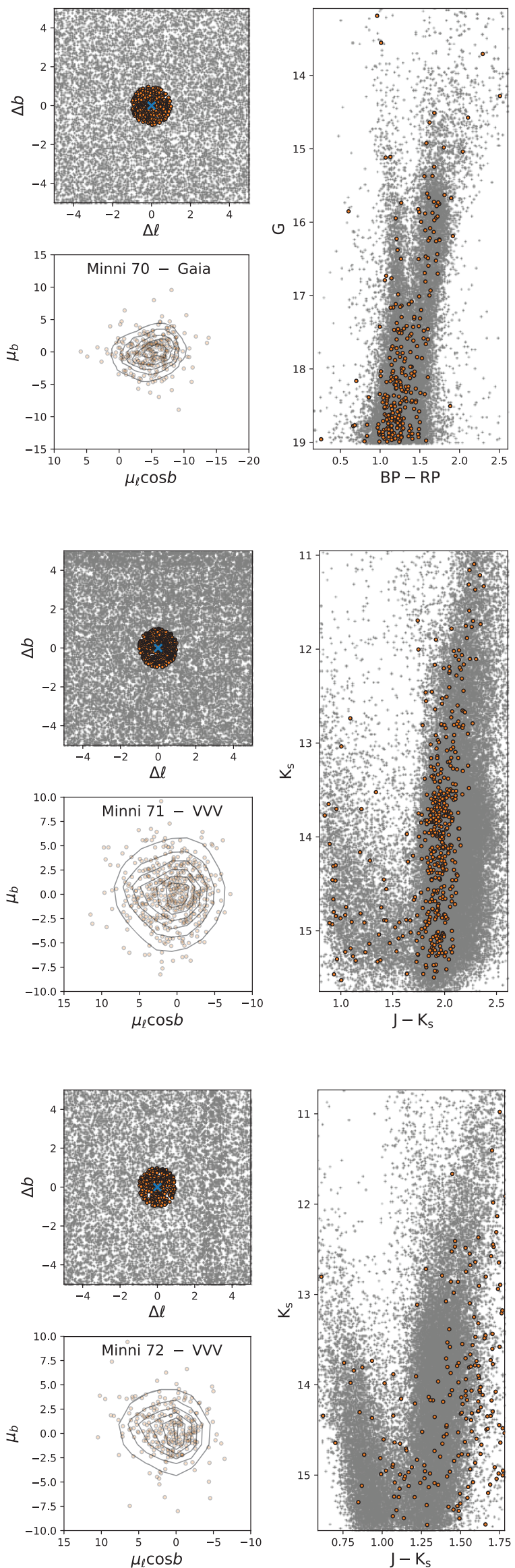

Fig. A.1. continued. 
F. Gran et al.: Gaia and VVV view of the last bulge globular cluster candidates
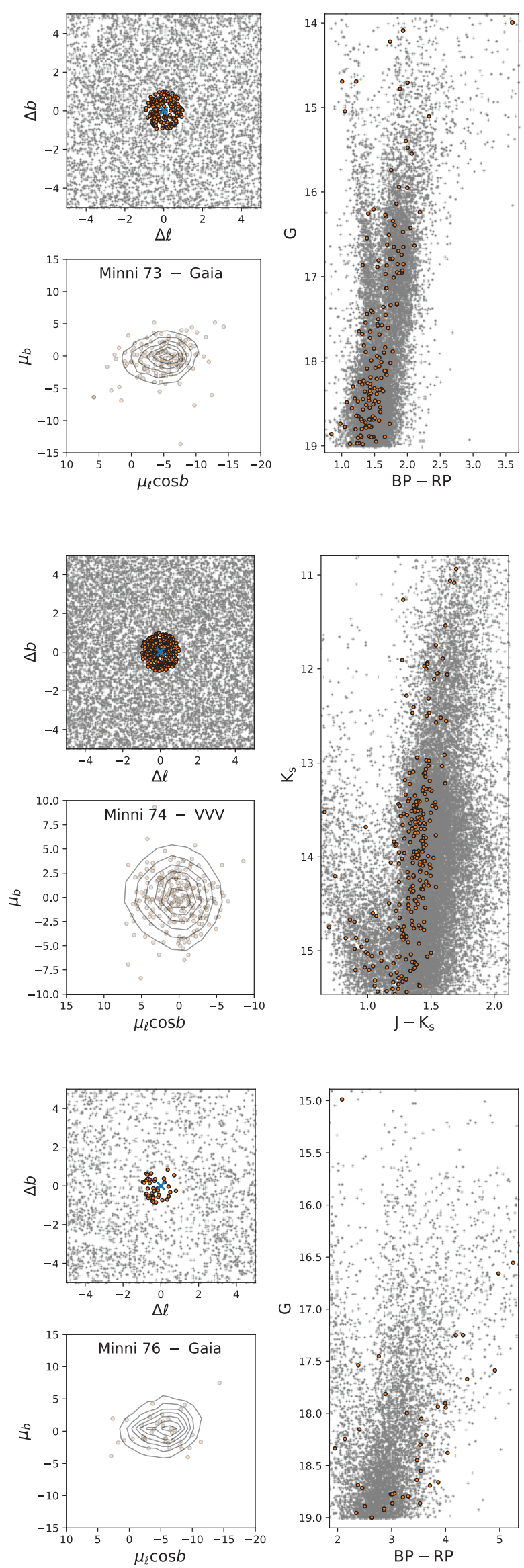
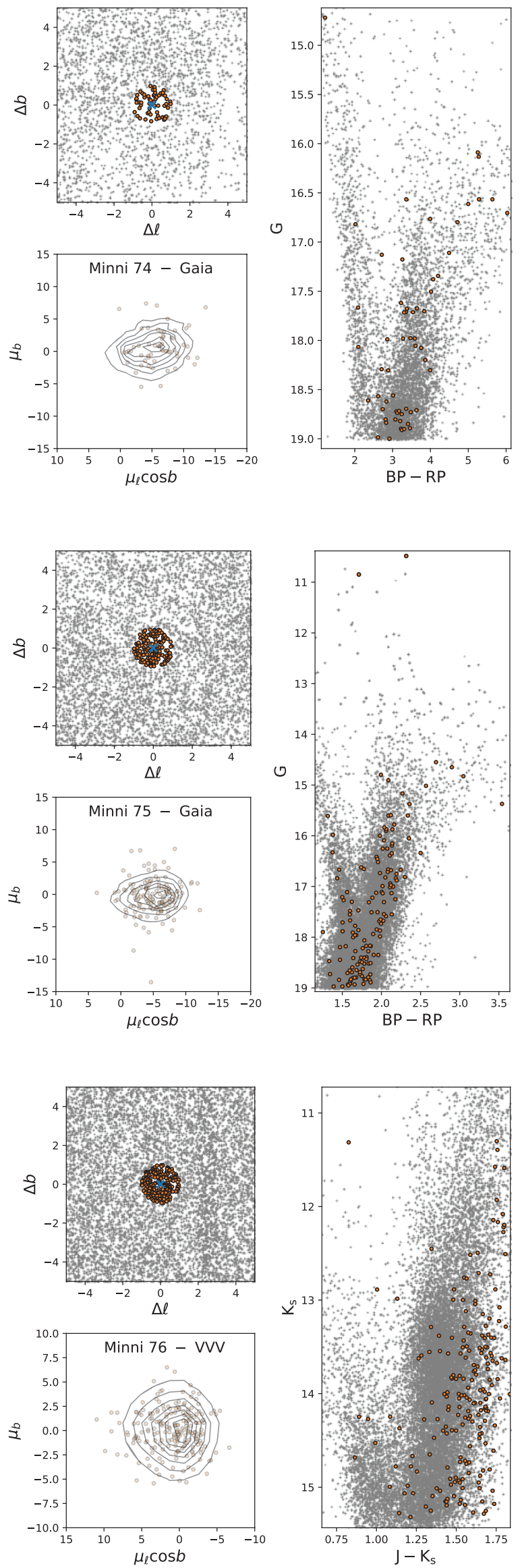

Fig. A.1. continued. 
A\&A 628, A45 (2019)
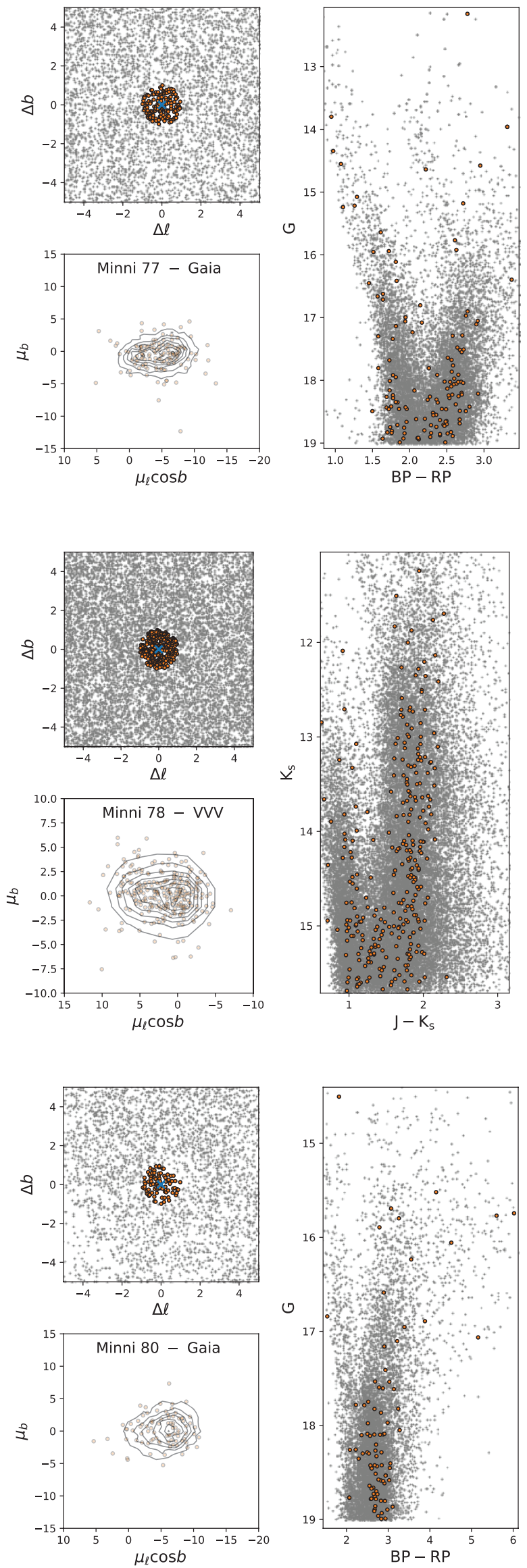
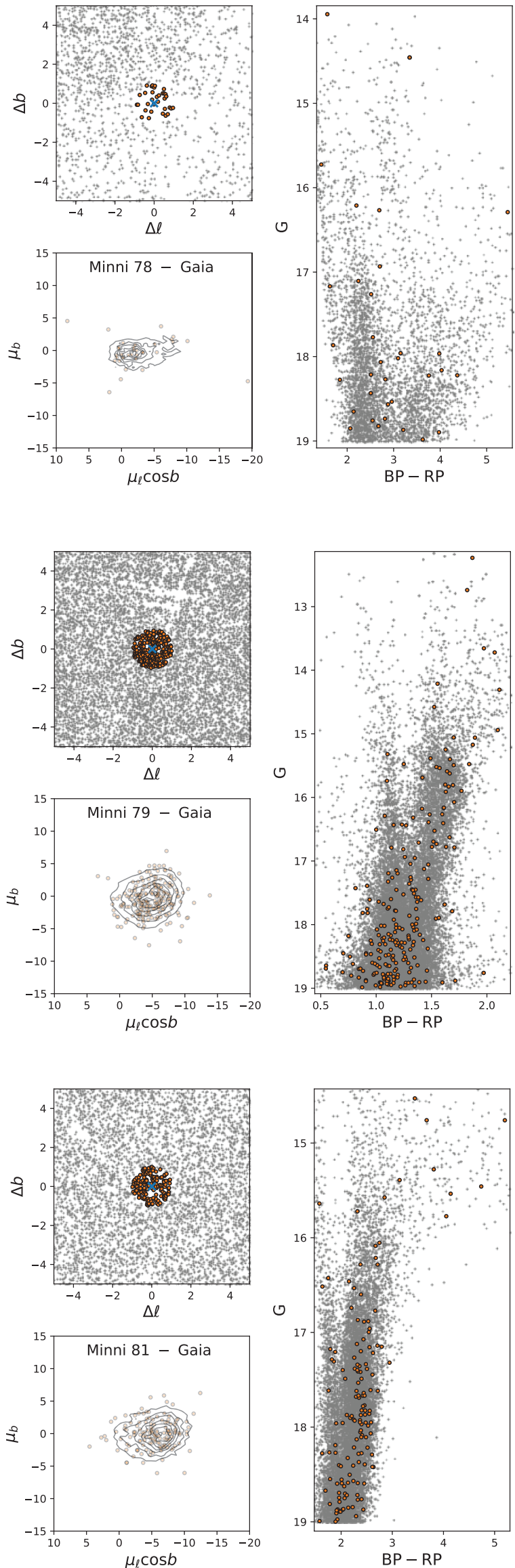

Fig. A.1. continued. 
F. Gran et al.: Gaia and VVV view of the last bulge globular cluster candidates
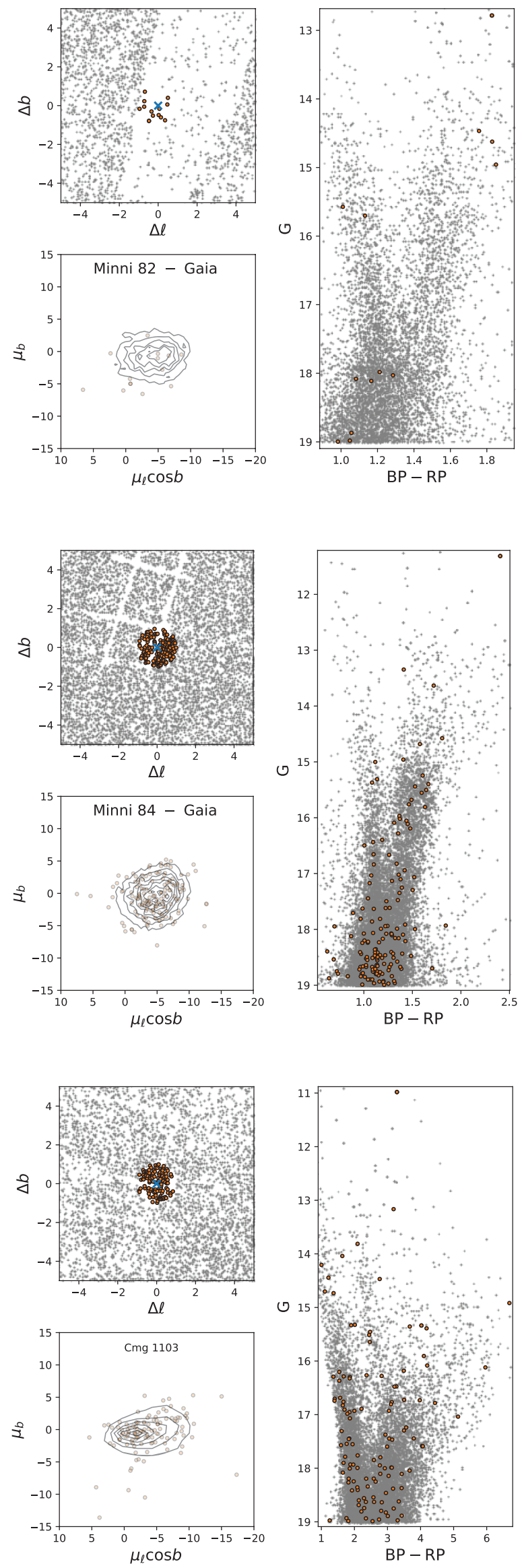
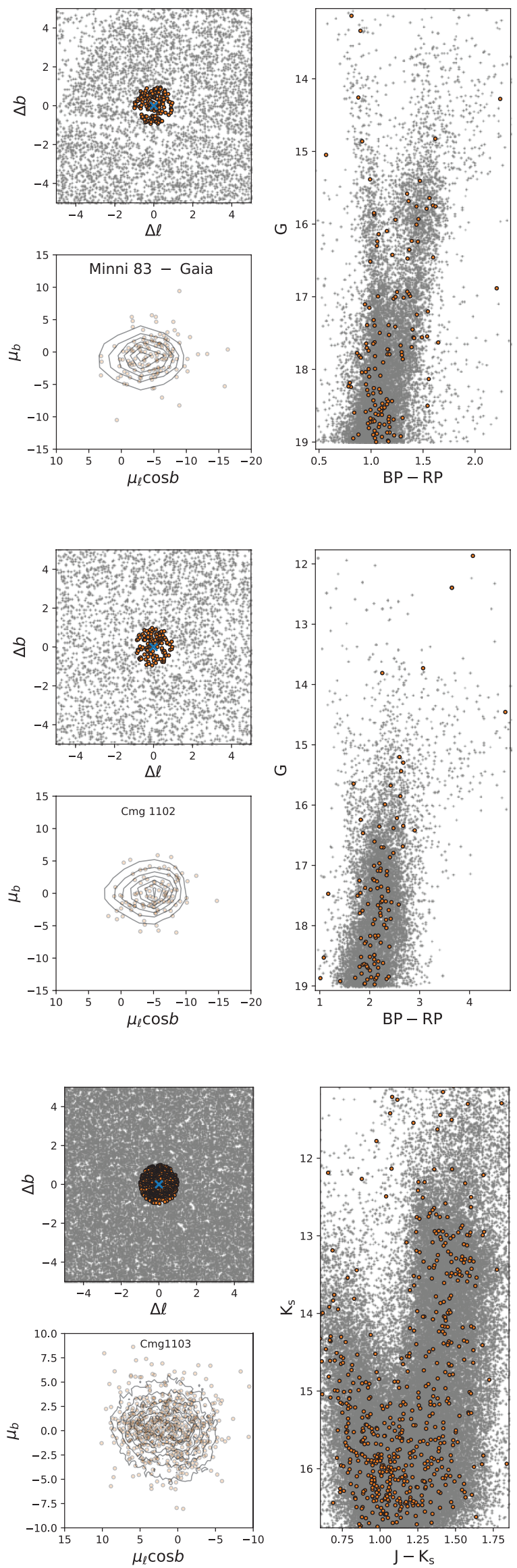

Fig. A.1. continued. 

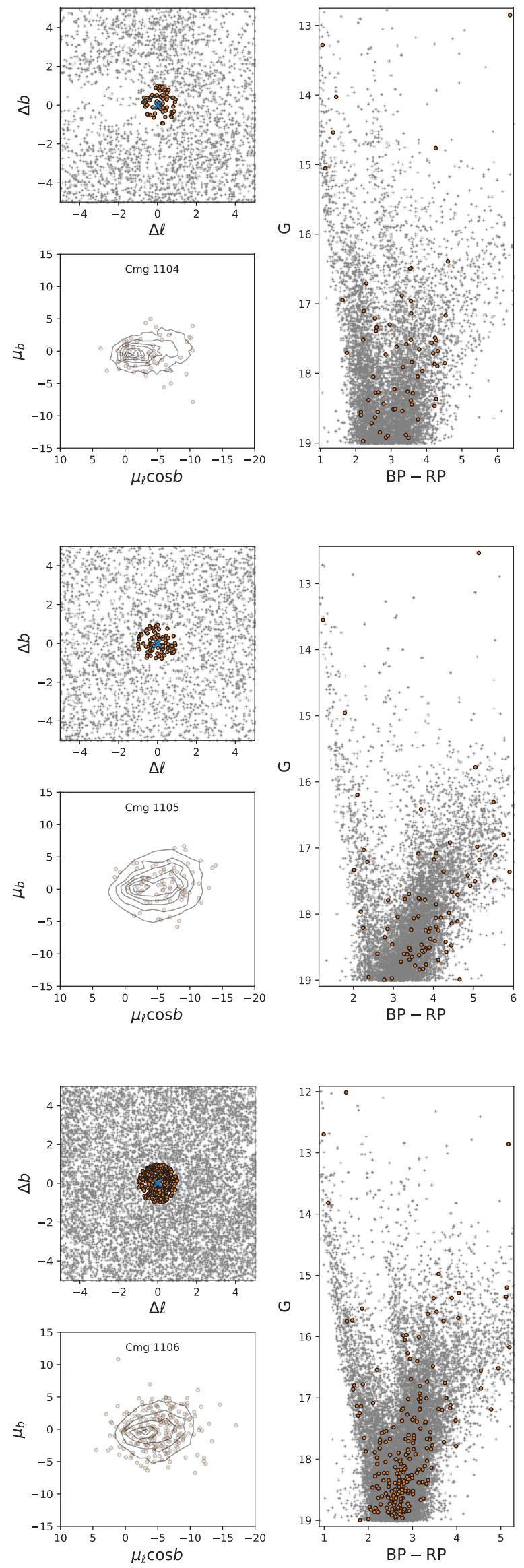
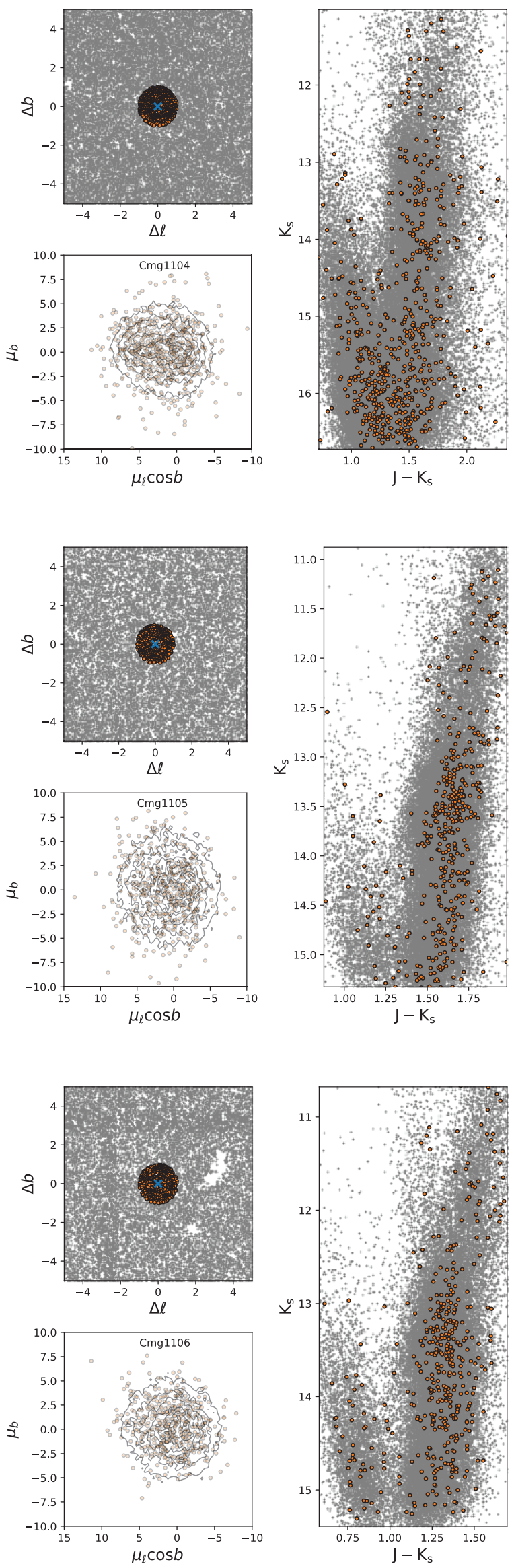

Fig. A.1. continued. 